\title{
Effect of Age on Clinical Outcomes Following On-/Off-Pump Coronary Artery Bypass: Meta- Analysis and Meta-Regression
}

\author{
Hayley Mauldon ${ }^{1}$, MD; Gudrun Dieberg ${ }^{2}$, MD; Neil Smart² ${ }^{2}$ MD; Nicola King ${ }^{1}$, MD
}

DOI: 10.21470/1678-9741-2018-0388

\begin{abstract}
Objective: There is currently much debate about which patients would benefit more after on- or off-pump coronary artery bypass grafting (CABG). The aim of this meta-analysis and meta-regression is to investigate the effect of age on short-term clinical outcomes after these approaches.

Methods: To identify potential studies, systematic searches were carried out in the Excerpta Medica dataBASE (EMBASE), PubMed, Web of Science, and the Cochrane Central Register of Controlled Trials (CENTRAL). The search strategy included the key concepts of "cardiopulmonary bypass" AND "coronary artery bypass grafting" AND "off pump" OR "on pump". This was followed by a meta-analysis and meta-regression investigating the effect of age on the incidences of stroke, myocardial infarction (MI), and mortality.

Results: Thirty-seven studies including 15,324 participants were analysed. Overall, there was a significant odds reduction
\end{abstract}

for patients receiving off-pump CABG suffering a stroke (odds ratio [OR] $0.770,95 \%$ confidence intervals $[\mathrm{Cl}] 0.594,0.998$, $P=0.048$ ); however, when patients were subdivided according to different age bands, this difference disappeared. There were also no significant differences in the odds of mortality (OR 0.876 , $95 \% \mathrm{Cl} 0.703,1.093, P=0.241$ ) or $\mathrm{MI}$ (OR $0.937,95 \% \mathrm{Cl} 0.795$, 1.105, $P=0.439$ ). Meta-regression analysis revealed no significant relationship between age and stroke $(P=0.652)$, age and mortality $(P=548)$, and age and $\mathrm{MI}(P=0.464)$.

Conclusion: Patients undergoing CABG are becoming older and may suffer from multiple comorbidities increasing their risk profile. However, with respect to short-term clinical outcomes, the patient's age does not help in determining whether off- or on-pump is superior.

Keywords: Cardiopulmonary Bypass. Coronary Artery Bypass, Off-Pump. Myocardial Infarction. Stroke. Comorbidity. Incidence.

\begin{tabular}{llll}
\hline \multicolumn{2}{l}{ Abbreviations, acronyms \& symbols } & & \\
\hline ARDS & $=$ Acute respiratory distress syndrome & MDA & $=$ Malondialdehyde \\
CABG & $=$ Coronary artery bypass grafting & MI & $=$ Myocardial infarction \\
CENTRAL & $=$ Cochrane Central Register of Controlled Trials & MRI & $=$ Magnetic resonance imaging \\
Cl & $=$ Confidence intervals & N/A & $=$ Not available. \\
CK-MB & $=$ Creatine kinase-muscle/brain & NR & $=$ Not reported \\
CMA & $=$ Comprehensive Meta-Analysis & NT-proBNP & $=$ N-terminal pro b-type natriuretic peptide \\
CPB & $=$ Cardiopulmonary bypass & OR & $=$ Odds ratio \\
CTnI & $=$ Cardiac troponin I & PRISMA & $=$ Preferred Reporting Items for Systematic Reviews \\
EMBASE & $=$ Excerpta Medica dataBASE & & and Meta-analyses \\
GOPCABE & $=$ German Off-Pump Coronary Artery Bypass & RCT & $=$ Randomised controlled trials \\
hs-CRP & Grafting in Elderly Patients & UK & $=$ United Kingdom \\
ICU & $=$ High-sensitivity creatine phosphate & USA & $=$ United States of America
\end{tabular}

'University of Plymouth, Ringgold Standard Institution, Plymouth, United Kingdom of Great Britain and Northern Ireland.

${ }^{2}$ University of New England, Ringgold Standard Institution, Armidale, Australia.

This study was carried out at the University of Plymouth, School of Biomedical Sciences, Faculty of Medicine and Dentistry, Plymouth, United Kingdom of Great Britain and Northern Ireland.
Correspondence Address:

Nicola King

(iD) https://orcid.org/0000-0001-6989-5760

University of Plymouth, School of Biomedical Sciences, Faculty of Health

Drake Circus, Plymouth PL48AA, United Kingdom of Great Britain and Northern Ireland

E-mail: nicola.king@plymouth.ac.uk 


\section{INTRODUCTION}

Coronary artery bypass grafting (CABG) is the gold standard treatment for patients with complex coronary artery disease. Originally in the 1950s, this surgery was carried out on-pump with cardiopulmonary bypass (CPB); however, this approach can be associated with aortic damage, myocardial ischaemic injury, renal damage, coagulation disorders, and systemic proinflammatory responses ${ }^{[1]}$. In addition, the use of side biting clamps can cause the embolization of atherosclerotic material leading to neurological events. To overcome these problems, off-pump CABG was introduced in the early 1960s, which reduces the amount of aortic manipulation. This approach has problems, the surgery is more technically challenging and there can be limitations associated with graft patency, completeness of revascularisation, and repeat revascularisation requirement ${ }^{[1]}$. The controversy as to which approach is superior has not been resolved by recent meta-analyses ${ }^{[2-4]}$.

Recently, a meta-analysis was published investigating the longterm outcomes of on- vs. off-pump CABG ${ }^{[5]}$. The accompanying editorial comment suggested that the discussion should be refocused from comparing each approach overall to investigating precisely which groups of patients would benefit more from which technique ${ }^{[6]}$. In this respect, one group of interest is elderly people. The age of patients undergoing CABG is continually rising as a result of an increasingly aged population and improved survival rates following diagnoses ${ }^{[7]}$. For example, Ozen et al. ${ }^{[8]}$ found out that octogenarians continue to have a higher morbidity and mortality rate following CABG than younger populations. Thus, highlighting the need for investigation into the most beneficial techniques within older generations.

Yuksel et al. ${ }^{[9]}$ studied patients with age of $>70$ years and concluded that there was no significant benefit of either technique in terms of postoperative complications and mortality. However, they did find out that off-pump CABG required significantly less transfused blood products. One of the largest studies to date that included 2,539 participants with 75 years or older was the German Off-Pump Coronary Artery Bypass Grafting in Elderly Patients (GOPCABE) trial[10]. Again, this study found no difference between off-pump and on-pump CABG in elderly patients in terms of mortality, stroke, or MI as well as repeat revascularisation or new renal-replacement therapy after surgery. There have also been three meta-analyses investigating the effects of on- vs. off-pump CABG in patients with age of $>70$ or $>80$ years. The results are contradictory, e.g., Altarabsheh et al. ${ }^{[11]}$ found higher rates of stroke following on-pump surgery, whilst Panesar et al..12] and Zhu et al. ${ }^{[13]}$ found comparable rates. Although elderly people represent an important subset of patients, there is a much broader age range of patients undergoing on- or off-pump CABG. Therefore, the aim of this novel meta-analysis is to investigate the effect of on- vs. offpump CABG on short-term clinical outcomes across the full age range of patients using both meta-analysis and meta-regression.

\section{METHODS}

This analysis was planned in accordance with the current guidelines for performing comprehensive systematic reviews and meta-analysis with meta-regression, including the Preferred Reporting Items for Systematic Reviews and Meta-analyses (PRISMA) guidelines ${ }^{[14]}$.

\section{Search Strategy}

To identify potential studies, systematic searches were carried out using the following databases: Excerpta Medica dataBASE (EMBASE), PubMed, Web of Science, and Cochrane Central Register of Controlled Trials (CENTRAL). The search was supplemented by scanning the reference lists of eligible studies. The search strategy included the key concepts of "cardiopulmonary bypass" AND "coronary artery bypass grafting" AND "off pump" OR "On pump" (Supplementary Figure 1). All identified papers were assessed independently by two reviewers (authors HM and NK). A third reviewer (author NS) was consulted to resolve disputes. Searches of published papers were conducted up until July 2018.

\section{Types of Studies Included}

This meta-analysis and meta-regression only included randomised controlled trials (RCT) comparing patients undergoing on- vs. off-pump CABG. There were no language restrictions. Animal studies, review papers, and non-randomised controlled trials were excluded. Studies that did not have any of the desired outcome measures or participants who were treated by other modalities, such as percutaneous coronary intervention, were excluded. Incomplete data or data from an already included study were excluded. Studies that included interventions other than off-pump vs. on-pump CABG were excluded. Studies where the mean ages of patients in each group were in different age bands were excluded. Studies where there were no mortality, strokes, or myocardial infarctions (MI) rates, leading to an incalculable odds ratio (OR), were excluded.

\section{Participants/Population}

This meta-analysis analysed RCTs of both male and female adult ( $\geq 18$ years old) patients with coronary artery disease who were undergoing either off- or on-pump CABG. Other treatment modalities and interventions for coronary artery disease, such as percutaneous coronary intervention, were excluded.

\section{Intervention(S), Exposure(S)}

This meta-analysis considered all RCTs where patients with stable angina or acute coronary syndrome were treated with either on-pump or off-pump CABG. More specifically, all RCTs where the intervention of carrying out $C A B G$ without the use of CPB were performed.

\section{Comparator(S)/Control}

The studies in this analysis compared off-pump CABG with a usual care control group receiving on-pump CABG.

\section{Search Results}

Our initial search found 2,161 articles. Of these, 2,074 studies were excluded based on title and abstract and 36 studies were 


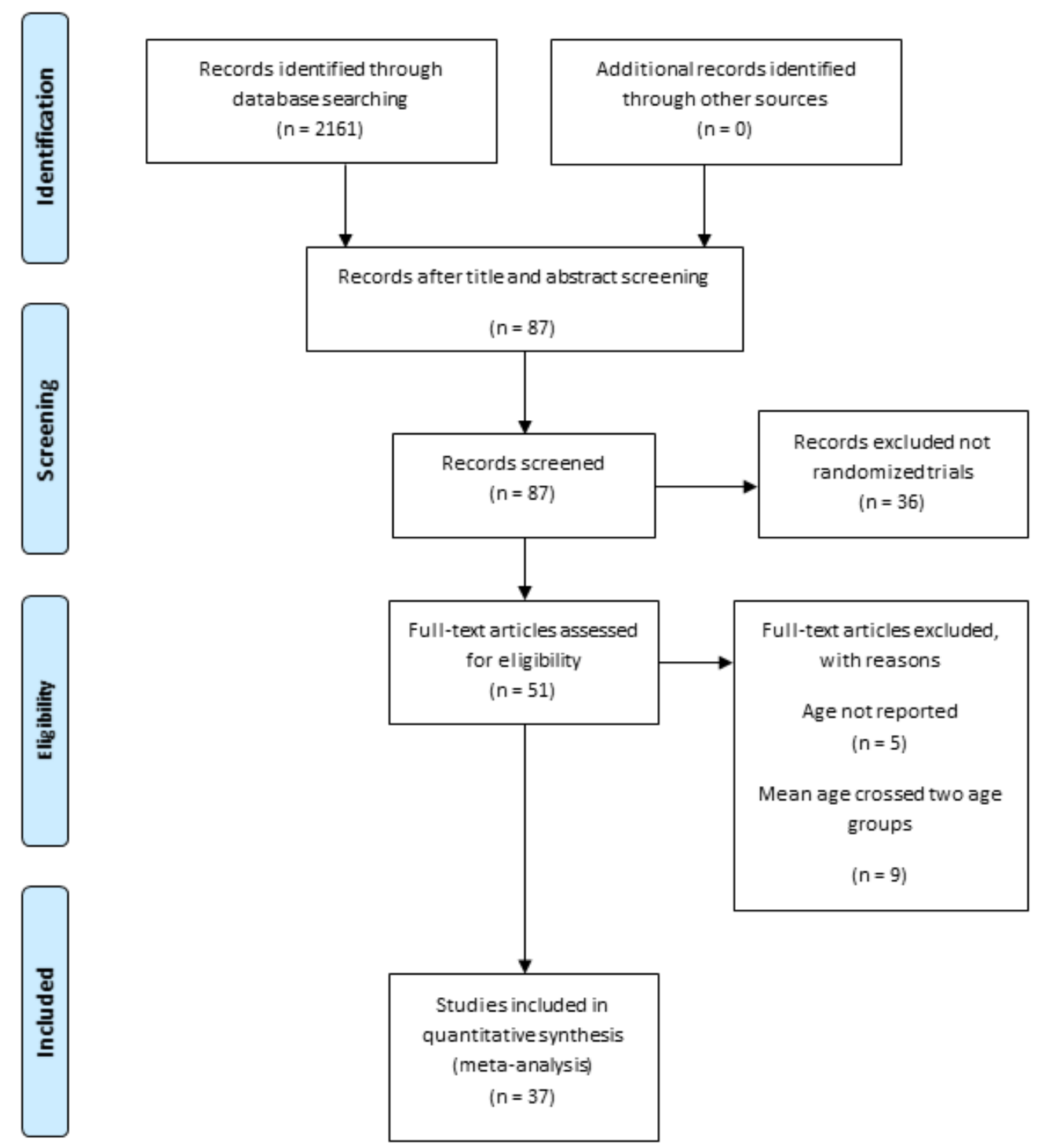

Fig. 1 - Consort figure. A flow diagram showing how the initial search results were refined until a group of studies that met all the inclusion criteria were found.

excluded as they were not RCTs. Of the RCTs, we excluded 14 studies, because either they had not reported the age of the patients or the mean age of the patients crossed two age bands (Figure 1). Thirtyseven studies were included in our analysis [S1-S37].

\section{Outcome(S)}

The primary outcomes analysed were short-term ( $<30$ days) incidences of stroke, mortality, and MI.

\section{Risk of Bias (Quality) Assessment}

Risk of bias was assessed using a modification of the Jadad scale ${ }^{[15]}$

\section{Strategy for Data Synthesis}

Data was collected by two authors and independently verified by a third author using pre-established tables. Patients were divided into 5-year age groups beginning at 51-55 and ending at 76-80 and investigated in their individual groups using subgroup analysis. All meta-analysis data was dichotomous and calculated as OR. An OR is a measure of association between an exposure and an outcome. The OR represents the odds that an outcome will occur given a particular exposure, compared to the odds of the outcome occurring in the absence of that exposure. Heterogeneity was quantified using the Cochrane Q test ${ }^{[16]}$, where $\mathrm{I}^{2}=0 \%$ represents no heterogeneity and $\mathrm{I}^{2}=100 \%$ represents considerable heterogeneity. A random-effects inverse 
variance model was used throughout. All meta-regression data was plotted as the log OR vs. the mean age of the patients in the off-pump group. In these graphs, a negative log OR favours off-pump and a positive log OR favours on-pump. We used a $5 \%$ level of significance and 95\% confidence intervals (Cl). All analyses were carried out in and all figures were produced in Comprehensive Meta-Analysis (CMA) V3.

\section{RESULTS}

The 37 studies included in the analysis had an aggregate of 15,324 participants, 7,661 of which had on-pump CABG and 7,663 had off-pump CABG. Table 1 summarises the characteristics of the included studies. Supplementary Table 1 lists the excluded $\mathrm{RCT}$ s and reasons for exclusion.

Table 1. Characteristics of the included studies.

\begin{tabular}{|c|c|c|c|c|c|}
\hline $\begin{array}{l}\text { Age range } \\
\text { (years) }\end{array}$ & Study & $\begin{array}{l}\text { N on CPB } \\
\text { (off CPB) }\end{array}$ & $\begin{array}{l}\text { Age on CPB } \\
\text { (off CPB) }\end{array}$ & $\begin{array}{l}\text { Male \% on CPB } \\
\text { (off CPB) }\end{array}$ & All outcome measures \\
\hline $51-55$ & $\begin{array}{c}\text { Iqbal et al. }{ }^{[23]}, 2014 \\
\text { Pakistan }\end{array}$ & $100(100)$ & $\begin{array}{c}53.5 \pm 10 \\
(51.6 \pm 10.3)\end{array}$ & NR & $\begin{array}{l}\text { Encephalopathy } \\
\text { Hospital stay } \\
\text { ICU stay } \\
\text { MI } \\
\text { Mortality } \\
\text { Renal failure } \\
\text { Stroke } \\
\text { Ventilation time }\end{array}$ \\
\hline \multirow[t]{4}{*}{$56-60$} & $\begin{array}{c}\text { Bicer et al.[24], } 2014 \\
\text { Turkey }\end{array}$ & $25(25)$ & $\begin{array}{l}56.9 \pm 10.7 \\
(57.7 \pm 8.4)\end{array}$ & $88(88)$ & $\begin{array}{l}\text { Mortality } \\
\text { MDA } \\
\text { hs-CRP } \\
\text { M30 } \\
\text { M65 }\end{array}$ \\
\hline & $\begin{array}{c}\text { Gerola et al. }{ }^{[25]}, 2004 \\
\text { Brazil }\end{array}$ & $80(80)$ & $\begin{array}{c}58.9 \pm 8.9 \\
(59.1 \pm 9.7)\end{array}$ & $68(64)$ & $\begin{array}{l}\text { Atrial fibrillation } \\
\text { CK-MB } \\
\text { Hospital stay } \\
\text { ICU stay } \\
\text { Ml } \\
\text { Mortality } \\
\text { Stroke }\end{array}$ \\
\hline & $\begin{array}{c}\text { Kobayashi et al. }{ }^{[26]} \\
\text { (JOCRI), } 2005 \\
\text { Japan }\end{array}$ & $86(81)$ & $\begin{array}{l}59 \pm 10 \\
(60 \pm 7)\end{array}$ & $86(87)$ & $\begin{array}{l}\text { Atrial fibrillation } \\
\text { CK-MB } \\
\text { Graft patency } \\
\text { ICU stay } \\
\text { MI } \\
\text { Mortality } \\
\text { Neuron specific enolase } \\
\text { S-100 protein } \\
\text { Stroke } \\
\text { Ventilation time }\end{array}$ \\
\hline & $\begin{array}{l}\text { Penttila et al. }{ }^{[27]}, 2001 \\
\text { Finland }\end{array}$ & $11(11)$ & $\begin{array}{l}59.2 \\
59.5\end{array}$ & NR & $\begin{array}{l}\text { Ml } \\
\text { Myocardial markers } \\
\text { Myocardial metabolism }\end{array}$ \\
\hline \multirow[t]{2}{*}{$61-65$} & $\begin{array}{c}\text { Al-Ruzzeh et al. }{ }^{[28]}, 2006 \\
\text { UK }\end{array}$ & $84(84)$ & $\begin{array}{l}63.1 \pm 9.6 \\
(63.1 \pm 11)\end{array}$ & $84(83)$ & $\begin{array}{l}\text { Atrial fibrillation } \\
\text { Blood transfusions } \\
\text { Graft patency } \\
\text { Health-related quality of life } \\
\text { Hospital stay } \\
\text { ICU stay } \\
\text { Mortality } \\
\text { Neurocognitive function } \\
\text { Stroke } \\
\text { Ventilation time }\end{array}$ \\
\hline & $\begin{array}{c}\text { Angelini et al. }{ }^{[29]}, 2002 \\
\text { UK }\end{array}$ & $\begin{array}{l}\text { BHACAS } 1100(100) \\
\text { BHACAS } 2101(100)\end{array}$ & $\begin{array}{c}\text { BHACAS } 161.7 \pm 8.6 \\
(62.2 \pm 9.6) \\
\text { BHACAS } 261.2 \pm 9.2 \\
\quad(63.8 \pm 8.5)\end{array}$ & $\begin{array}{l}\text { BHACAS } 179(82) \\
\text { BHACAS } 285(82)\end{array}$ & $\begin{array}{l}\text { Atrial fibrillation } \\
\text { MI } \\
\text { Mortality } \\
\text { Stroke }\end{array}$ \\
\hline
\end{tabular}




\begin{tabular}{|c|c|c|c|c|}
\hline $\begin{array}{c}\text { Ascione et al.[30], } 2000 \\
\text { UK }\end{array}$ & $100(100)$ & $63(63)$ & $79(82)$ & $\begin{array}{l}\text { Atrial fibrillation } \\
\text { Hospital stay } \\
\text { ICU stay } \\
\text { MI } \\
\text { Mortality } \\
\text { Stroke } \\
\text { Ventilation time }\end{array}$ \\
\hline $\begin{array}{c}\text { Fattouch et al. }{ }^{[31]}, 2009 \\
\text { Italy }\end{array}$ & $65(63)$ & $\begin{array}{c}61 \pm 18 \\
(63 \pm 16)\end{array}$ & $77(61)$ & $\begin{array}{l}\text { CTnl } \\
\text { Cardiac contractile function } \\
\text { Hospital stay } \\
\text { ICU stay } \\
\text { Mortality } \\
\text { Ventilation time }\end{array}$ \\
\hline $\begin{array}{c}\text { Jongman et al.[32], } 2014 \\
\text { The Netherlands }\end{array}$ & $29(30)$ & $\begin{array}{l}63 \\
(63)\end{array}$ & $90(90)$ & $\begin{array}{l}\text { Cardiac failure } \\
\text { Inflammatory markers } \\
\text { Major bleeding } \\
\text { Pulmonary embolism } \\
\text { Renal failure } \\
\text { Stroke }\end{array}$ \\
\hline $\begin{array}{c}\text { Khan et al. }{ }^{[33]}, 2004 \\
\text { UK }\end{array}$ & $50(54)$ & 64.7 & $82(93)$ & $\begin{array}{l}\text { Blood loss } \\
\text { Extubation time } \\
\text { Hospital stay } \\
\text { ICU stay } \\
\text { Infection } \\
\text { Low cardiac output } \\
\text { MI } \\
\text { Mortality } \\
\text { Repeat surgery }\end{array}$ \\
\hline $\begin{array}{l}\text { Kok et al. }{ }^{[34]}, 2014 \\
\text { The Netherlands }\end{array}$ & $29(30)$ & $\begin{array}{c}62.6 \pm 9.9 \\
(63 \pm 9)\end{array}$ & $90(90)$ & $\begin{array}{l}\text { Cerebral oxygenation } \\
\text { Cognitive dysfunction } \\
\text { Hospital stay } \\
\text { ICU stay } \\
\text { Stroke }\end{array}$ \\
\hline $\begin{array}{l}\text { Légaré et al. }{ }^{[35]}, 2004 \\
\text { Canada }\end{array}$ & $150(150)$ & $\begin{array}{c}63.7 \pm 10 \\
(62.1 \pm 10.1)\end{array}$ & $79(81)$ & $\begin{array}{l}\text { Atrial fibrillation } \\
\text { Hospital stay } \\
\text { ICU stay } \\
\text { MI } \\
\text { Mortality } \\
\text { Stroke } \\
\text { Transfusion requirement } \\
\text { Ventilation time } \\
\text { Wound infection }\end{array}$ \\
\hline $\begin{array}{l}\text { Lingaas et al. }{ }^{[36]}, 2004 \\
\text { Norway }\end{array}$ & $60(60)$ & $\begin{array}{l}65 \pm 8.3 \\
64 \pm 7.8\end{array}$ & $72(85)$ & $\begin{array}{l}\text { CPB time } \\
\text { Ventilation time } \\
\text { Reintubation } \\
\text { Bleeding } \\
\text { Blood transfusions } \\
\text { Atrial fibrillation } \\
\text { CK-MB } \\
\text { Aspartate aminotransferase } \\
\text { Stroke } \\
\text { Mortality } \\
\text { Mediastinitis } \\
\text { Graft patency } \\
\end{array}$ \\
\hline $\begin{array}{c}\text { Lund et al.[37], } 2003 \\
\text { Norway }\end{array}$ & $22(29)$ & $64(62)$ & $73.9(89.7)$ & $\begin{array}{l}\text { Cerebral blood flow } \\
\text { Cerebral MRI } \\
\text { Neuropsychologic tests } \\
\text { Stroke }\end{array}$ \\
\hline $\begin{array}{l}\text { Michaux et al. }{ }^{[38]}, 2011 \\
\text { Switzerland }\end{array}$ & $25(25)$ & $\begin{array}{c}65 \pm 8 \\
(61 \pm 9)\end{array}$ & $84(84)$ & $\begin{array}{l}\text { Atrial fibrillation } \\
\text { CTnl } \\
\text { Hospital stay } \\
\text { ICU stay } \\
\text { Ml } \\
\text { Mortality } \\
\text { Right ventricular function } \\
\text { Ventilation time }>12 \text { hours }\end{array}$ \\
\hline
\end{tabular}




\begin{tabular}{|c|c|c|c|c|}
\hline $\begin{array}{c}\text { Motallebzdah et al. }{ }^{[39]}, \\
2004 \\
\text { UK }\end{array}$ & $20(15)$ & $63(65)$ & $90(93)$ & $\begin{array}{l}\text { Cerebral blood flow } \\
\text { S100 protein } \\
\text { Stroke }\end{array}$ \\
\hline $\begin{array}{c}\text { Motallebzdah et al. }{ }^{[40]}, \\
2007 \\
\text { UK }\end{array}$ & $104(108)$ & $\begin{array}{c}65.1 \pm 0.9 \\
(63.9 \pm 0.9)\end{array}$ & 91 (87) & $\begin{array}{l}\text { Cerebral emboli } \\
\text { Mortality } \\
\text { Neurocognitive function } \\
\text { Stroke }\end{array}$ \\
\hline $\begin{array}{l}\text { Nathoe et al. }{ }^{[41]} \\
\text { (Octopus), } 2003 \\
\text { USA }\end{array}$ & 139 (142) & $\begin{array}{l}60.8 \pm 8.8 \\
61.7 \pm 9.2\end{array}$ & $71(66)$ & $\begin{array}{l}\text { Cost } \\
\text { Ml } \\
\text { Mortality } \\
\text { Quality of life } \\
\text { Repeat revascularisation } \\
\text { Stroke }\end{array}$ \\
\hline $\begin{array}{l}\text { Puskas et al. }{ }^{[42]} \\
\text { (SMART), } 2003 \text { USA }\end{array}$ & 99 (98) & $\begin{array}{c}62.5 \pm 9.5 \\
(62.2 \pm 11.1)\end{array}$ & 77 (78) & $\begin{array}{l}\text { Atrial fibrillation } \\
\text { Coagulopathy and transfusion } \\
\text { Hospital stay } \\
\text { ICU stay } \\
\text { MI } \\
\text { Mortality } \\
\text { Stroke }\end{array}$ \\
\hline $\begin{array}{l}\text { Rastan et al. }{ }^{[43]}, 2005 \\
\text { Germany }\end{array}$ & $20(20)$ & $\begin{array}{c}65.3 \pm 3.9 \\
(63 \pm 6)\end{array}$ & $80(80)$ & $\begin{array}{l}\text { CK-MB } \\
\text { C-reactive protein } \\
\text { CTnl } \\
\text { Intraoperative myocardial } \\
\text { ischaemia } \\
\text { Ml } \\
\text { Mortality } \\
\text { NT-proBNP } \\
\text { Oxidative stress } \\
\text { Stroke }\end{array}$ \\
\hline $\begin{array}{c}\text { Sahlman et al. }{ }^{[44]}, 2003 \\
\text { Finland }\end{array}$ & $26(24)$ & $\begin{array}{c}61.5 \pm 8.1 \\
(64 \pm 9)\end{array}$ & 77 (88) & $\begin{array}{l}\text { Extubation time } \\
\text { Bleeding } \\
\text { CK-MB } \\
\text { ICU stay } \\
\text { Hospital stay } \\
\text { Weight gain } \\
\text { Complement C3 C4 } \\
\text { Protein carbonyls } \\
\text { Wound infection } \\
\text { Low cardiac output syndrome } \\
\text { Cerebral infarction } \\
\text { Oxidative stress markers }\end{array}$ \\
\hline $\begin{array}{l}\text { Shroyer et al. }{ }^{[45]} \\
\text { (ROOBY), } 2009 \\
\text { USA }\end{array}$ & 1099 (1104) & $\begin{array}{l}62.5 \pm 8.5 \\
(63 \pm 8.5)\end{array}$ & 99 (99) & $\begin{array}{l}\text { Cardiac arrest } \\
\text { Coma } \\
\text { Hospital stay } \\
\text { ICU stay } \\
\text { Mediastinitis } \\
\text { Mortality } \\
\text { New mechanical support } \\
\text { Renal failure } \\
\text { Reoperation } \\
\text { Stroke } \\
\text { Tracheostomy } \\
\text { Ventilation time }\end{array}$ \\
\hline $\begin{array}{l}\text { Straka et al. }{ }^{[46]} \\
\text { (PRAGUE-4), } 2004 \\
\text { Czech Republic }\end{array}$ & $184(204)$ & $62(63)$ & $86(77)$ & $\begin{array}{l}\text { Atrial fibrillation } \\
\text { Hospital stay } \\
\text { ICU stay } \\
\text { MI } \\
\text { Mortality } \\
\text { Renal failure } \\
\text { Stroke } \\
\text { Ventilation time }\end{array}$ \\
\hline
\end{tabular}




\begin{tabular}{|c|c|c|c|c|c|}
\hline & $\begin{array}{l}\text { Vedin et al. } .^{[47]}, 2006^{[47]} \\
\text { Sweden }\end{array}$ & 37 (33) & $65(65)$ & 84 (78) & $\begin{array}{l}\text { Anxiety } \\
\text { Cognitive function } \\
\text { Depression } \\
\text { Ml } \\
\text { Stroke }\end{array}$ \\
\hline \multirow[t]{6}{*}{$66-70$} & $\begin{array}{l}\text { Carrier et al. }{ }^{[48]}, 2003 \\
\text { Canada }\end{array}$ & $37(28)$ & $\begin{array}{c}70 \pm 6 \\
(70 \pm 8)\end{array}$ & $84(68)$ & $\begin{array}{l}\text { Mortality } \\
\text { Ml } \\
\text { Stroke } \\
\text { Renal insufficiency } \\
\text { Respiratory failure/infection } \\
\text { Bleeding } \\
\text { Blood transfusions } \\
\text { ICU stay } \\
\text { Hospital stay }\end{array}$ \\
\hline & $\begin{array}{c}\text { Lamy et al }{ }^{[49]} \\
\text { (CORONARY), } 2012 \\
\text { Canada }\end{array}$ & 2377 (2375) & $\begin{array}{c}67.5 \pm 6.9 \\
(67.6 \pm 6.7)\end{array}$ & $82(80)$ & $\begin{array}{l}\text { Atrial fibrillation } \\
\text { Ml } \\
\text { Mortality } \\
\text { New renal failure } \\
\text { Stroke }\end{array}$ \\
\hline & Lee et al ${ }^{[50]}, 2003$ Hawaii & $30(30)$ & $\begin{array}{c}66 \pm 11.2 \\
(65.5 \pm 9.6)\end{array}$ & $73(80)$ & $\begin{array}{l}\text { Cerebral microemboli } \\
\text { Cerebral perfusion } \\
\text { Cost } \\
\text { Hospital stay } \\
\text { Mortality } \\
\text { Neurological function } \\
\text { Stroke }\end{array}$ \\
\hline & $\begin{array}{l}\text { Muneretto et al. }{ }^{[51]}, 2003 \\
\text { Italy }\end{array}$ & $88(88)$ & $\begin{array}{c}66 \pm 9 \\
(67 \pm 8)\end{array}$ & $59(63)$ & $\begin{array}{l}\text { Abdominal infarction } \\
\text { Atrial fibrillation } \\
\text { Hospital stay } \\
\text { ICU stay } \\
\text { Ml } \\
\text { Mortality } \\
\text { Stroke } \\
\text { Ventilation time }\end{array}$ \\
\hline & $\begin{array}{l}\text { Nesher et al. }{ }^{[52]}, 2006 \\
\text { Israel }\end{array}$ & $60(60)$ & $\begin{array}{c}68 \pm 5 \\
(67 \pm 1)\end{array}$ & $77(73)$ & $\begin{array}{l}\text { CK-MB } \\
\text { cTnl } \\
\text { Cytokines } \\
\text { Hospital stay } \\
\text { Stroke } \\
\text { Ventilation time } \\
\end{array}$ \\
\hline & $\begin{array}{l}\text { Niranjan et al. }{ }^{[53]}, 2006 \\
\text { UK }\end{array}$ & $40(40)$ & $\begin{array}{c}66.3 \pm 7.3 \\
67.3 \pm 11.2\end{array}$ & $\begin{array}{l}80 \\
75\end{array}$ & $\begin{array}{l}\text { Atrial fibrillation } \\
\text { Blood transfusion requirements } \\
\text { Clotting tests } \\
\text { Hospital stay } \\
\text { ICU stay } \\
\text { Mortality } \\
\text { Postoperative blood loss } \\
\text { Stroke } \\
\text { Ventilation time }\end{array}$ \\
\hline \multirow[t]{3}{*}{ 71-75 } & $\begin{array}{l}\text { Hlavicka et al. }{ }^{[54]} \\
\text { (PRAGUE-6), } 2016 \\
\text { Czech Republic }\end{array}$ & 108 (98) & $\begin{array}{l}73.6 \pm 7.4 \\
74.7 \pm 6.5\end{array}$ & $57.4(59.2)$ & $\begin{array}{l}\text { Ml } \\
\text { Mortality } \\
\text { Renal failure } \\
\text { Stroke }\end{array}$ \\
\hline & $\begin{array}{c}\text { Houlind et al. }{ }^{[55]} \text { (DOORS), } \\
2012 \\
\text { Denmark }\end{array}$ & $450(450)$ & $75(75)$ & $78(76)$ & $\begin{array}{l}\text { Hospital stay } \\
\text { ICU stay } \\
\text { MI } \\
\text { Mortality } \\
\text { Quality of life } \\
\text { Stroke }\end{array}$ \\
\hline & $\begin{array}{l}\text { Lemma et al. }{ }^{[56]} \text { (ON-OFF), } \\
2012 \\
\text { Italy }\end{array}$ & 203 (208) & $73(74)$ & $69(70)$ & $\begin{array}{l}\text { MI } \\
\text { Mortality } \\
\text { Renal failure } \\
\text { Stroke } \\
\text { Reoperation for bleeding } \\
\text { ARDS }\end{array}$ \\
\hline
\end{tabular}




\begin{tabular}{|c|c|c|c|c|c|}
\hline \multirow[t]{3}{*}{$76-80$} & $\begin{array}{c}\text { Diegeler et al. }{ }^{[10]} \\
\text { (GOPCABE), } 2013 \\
\text { Germany }\end{array}$ & 1207 (1187) & $\begin{array}{c}78.4 \pm 2.9 \\
(78.6 \pm 3.0)\end{array}$ & $68(69)$ & $\begin{array}{l}\text { Hospital stay } \\
\text { ICU stay } \\
\text { MI } \\
\text { Mortality } \\
\text { New renal-replacement therapy } \\
\text { Repeat revascularisation } \\
\text { Stroke } \\
\text { Ventilation time }\end{array}$ \\
\hline & $\begin{array}{l}\text { Møller et al. }{ }^{[57]} \\
\text { (BBS), } 2010 \\
\text { Denmark }\end{array}$ & $163(176)$ & $\begin{array}{c}75.6 \pm 4.9 \\
(76.1 \pm 5.2)\end{array}$ & $64(65)$ & $\begin{array}{l}\text { Cardiac arrest with successful } \\
\text { resuscitation } \\
\text { Coronary reintervention } \\
\text { Low cardiac output syndrome } \\
\text { MI } \\
\text { Mortality } \\
\text { Stroke }\end{array}$ \\
\hline & $\begin{array}{c}\text { Rogers et al. }{ }^{[58]} \text { (CRISP), } \\
2014 \\
\text { UK }\end{array}$ & $53(53)$ & $\begin{array}{c}75.7 \pm 7.7 \\
(76.4 \pm 5.8)\end{array}$ & $76(78)$ & $\begin{array}{l}\text { Ml } \\
\text { Mortality } \\
\text { Prolonged initial ventilation } \\
\text { Renal failure } \\
\text { Sternal wound dehiscence } \\
\text { Stroke }\end{array}$ \\
\hline
\end{tabular}

ARDS=acute respiratory distress syndrome; CK-MB=creatine kinase-muscle/brain; CPB=cardiopulmonary bypass; $C T n l=c a r d i a c$ troponin l; hs-CRP=highsensitivity creatine phosphate; $\mid \mathrm{CU}=$ =intensive care unit; $\mathrm{MDA}=$ malondialdehyde; $\mathrm{Ml}=$ =myocardial infarction; $\mathrm{MRI=magnetic}$ resonance imaging; $\mathrm{NR=not}$ reported; NT-proBNP=N-terminal pro b-type natriuretic peptide; UK=United Kingdom; USA=United States of America

\section{Stroke Incidence}

A total of 31 studies investigated the incidence of stroke. The overall OR was $0.770\left(95 \% \mathrm{Cl} 0.594,0.998, \mathrm{I}^{2}=0 \%, P=0.048\right)$. When the patients were grouped according to age, there were no significant differences in the odds of a stroke occurring in the off-pump group compared to the on-pump group. Fifty-one to 55 years old OR 0.32 ( $\left.95 \% \mathrm{Cl} 0.063,1.624, \mathrm{I}^{2}=0 \%, P=0.169\right)$; $56-60$ OR $0.203\left(95 \% \mathrm{Cl} 0.023,1.834, \mathrm{I}^{2}=0 \%, P=0.156\right) ; 61-65$ OR 0.884 (95\% Cl 0.522, 1.497, $\left.\left.\right|^{2}=0 \%, P=0.647\right) ; 66-70$ OR $0.801(95 \% \mathrm{Cl}$ $\left.0.486,1.321, I^{2}=0 \%, P=0.385\right) ; 71-75$ OR 0.555 (95\% Cl 0.275, 1.120, $\left.P^{2}=0 \%, P=0.100\right)$; and $76-80$ OR $0.879\left(95 \% \mathrm{Cl} 0.552,1.399, P^{2}=0\right.$, $P=0.586)$. See Figure 2 for the forest plot.

\section{Mortality Incidence}

A total of 27 studies investigated the mortality incidence. The overall OR was $0.876\left(95 \% \mathrm{Cl} 0.703,1.093, \mathrm{I}^{2}=0 \%, P=0.241\right)$. There was no significant difference in the odds of mortality occurring in the off-pump group compared to the on-pump group. This was also true when mortality was calculated according to different age groups. Fifty-one to 55 years old OR $0.660(95 \% \mathrm{Cl}$ $\left.0.108,4.036, I^{2}=0 \%, P=0.653\right)$; 56-60 OR 0.323 (95\% Cl 0.050, 2.096, $\left.\mathrm{P}^{2}=0 \%, P=0.236\right) ; 61-65$ OR $1.192\left(95 \% \mathrm{Cl} 0.717,1.980, \mathrm{I}^{2}=0 \%\right.$, $P=0.499) ; 66-70$ OR 0.889 (95\% Cl 0.634, 1.247, $\left.I^{2}=0 \%, P=0.495\right)$; 71-75 OR 0.722 ( $\left.95 \% \mathrm{Cl} 0.368,1.417, \mathrm{P}^{2}=0 \%, P=0.344\right)$; and $76-80$ OR $0.793\left(95 \% \mathrm{Cl} 0.511,1.231, I^{2}=0 \%, P=0.301\right)$. See Figure 3 for the forest plot.

\section{Myocardial Infarction Incidence}

A total of 28 studies investigated the Ml incidence. The overall OR was 0.937 ( $\left.95 \% \mathrm{Cl} 0.795,1.105, \mathrm{I}^{2}=0 \%, P=0.439\right)$. There was no difference in the odds of a MI happening in the off-pump group compared to the on-pump group. There was one significant result when patients were grouped according to age band. Fifty-one to 55 years old OR $6.056\left(95 \% \mathrm{Cl} 1.307,28.073, \mathrm{I}^{2}=0 \%\right.$, $P=0.021) ; 56-60$ OR 0.670 (95\% Cl 0.229, 1.962, $\left.I^{2}=0 \%, P=0.465\right)$; 61-65 OR $0.937\left(95 \% \mathrm{Cl} 0.627,1.401, \mathrm{I}^{2}=0 \%, P=0.753\right) ; 66-70 \mathrm{OR}$ $0.921\left(95 \% \mathrm{Cl} 0.737,1.151, \mathrm{I}^{2}=0 \%, P=0.469\right) ; 71-75$ OR 1.078 (95\% $\left.\mathrm{Cl} 0.689,1.688,\left.\right|^{2}=70 \%, P=0.742\right) ;$ and $76-80$ OR $0.763(95 \% \mathrm{Cl}$ $0.467,1.245, I^{2}=0 \%, P=0.279$ ). See Figure 4 for the forest plot.

\section{Risk of Bias}

Risk of bias was assessed using a modified Jadad scale with a maximum score of six (Supplementary Table 2). The median score was three. Publication bias was investigated using funnel plots, all of which were symmetrical. The funnel plots with their respective Begg and Mazumdar's test and Egger's test statistics can be found in Supplementary Figures 2 to 4 .

\section{Meta-Regression Analyses}

Figure 5 shows the meta-regression plot graphing the log of the OR for stroke occurrence against the mean age of the patients in the off-pump group. The regression line lies slightly on the side favouring off-pump, although the upper $95 \% \mathrm{Cl}$ lies on the side favouring on-pump. There is no difference in the modality favoured across the different ages measured and no relationship between age and the $\log \mathrm{OR}(\mathrm{Q}=0.200, P=0.652)$.

Figure 6 shows the meta-regression plot graphing the log of the OR for mortality occurrence against the mean age of the patients in the off-pump group. The meta-regression line begins on the side favouring on-pump and then moves to the side favouring off-pump as age increases; however, the $95 \% \mathrm{Cl}$ are equally dispersed either the side of the line of no effect across all the ages. Therefore, there is no difference in the modality favoured across the different ages measured and no relationship between age and the $\log \mathrm{OR}(\mathrm{Q}=0.360, P=0.548)$. 


\begin{tabular}{|c|c|c|c|c|c|}
\hline \multirow{2}{*}{$\frac{\text { Q pup by }}{\text { Aqpornos }}$} & \multirow[t]{2}{*}{ Study neme } & \multicolumn{4}{|c|}{ siti stos for sooh study } \\
\hline & & $\underset{\text { rxto }}{\text { Odds }}$ & $\begin{array}{l}\text { Lower } \\
\text { limt }\end{array}$ & $\begin{array}{l}\text { Upper } \\
\text { ilmit }\end{array}$ & p-Value \\
\hline $\begin{array}{l}5155 \\
5155\end{array}$ & 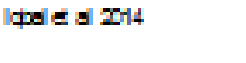 & $\begin{array}{l}0300 \\
0300\end{array}$ & $\begin{array}{l}0.083 \\
0.083\end{array}$ & $\begin{array}{l}1.804 \\
1.804\end{array}$ & $\begin{array}{l}0.1 \oplus 9 \\
0.1 \oplus 9\end{array}$ \\
\hline $\begin{array}{l}5600 \\
5690\end{array}$ & $\begin{array}{l}\text { Bcas es } 12014 \\
\text { Jock } 205\end{array}$ & $\begin{array}{l}0.125 \\
0.350\end{array}$ & $\begin{array}{l}0.006 \\
0.014\end{array}$ & $\begin{array}{l}255 \\
8 \pi 8\end{array}$ & $\begin{array}{l}0.178 \\
0.522\end{array}$ \\
\hline $\begin{array}{l}5690 \\
6105 \\
6105\end{array}$ & $\begin{array}{l}\text { ARmen es } 2006 \\
\text { BNCAB } 1200\end{array}$ & $\begin{array}{l}0.203 \\
1.000 \\
0.00\end{array}$ & $\begin{array}{l}0.003 \\
0.138 \\
0.108\end{array}$ & $\begin{array}{l}1.384 \\
7200 \\
4.036\end{array}$ & $\begin{array}{l}0.156 \\
1000 \\
0.653\end{array}$ \\
\hline $\begin{array}{l}6165 \\
6165\end{array}$ & 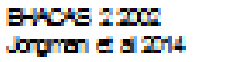 & $\begin{array}{l}0330 \\
0320\end{array}$ & $\begin{array}{l}0.054 \\
0.013\end{array}$ & $\begin{array}{l}3207 \\
3226\end{array}$ & $\begin{array}{l}0.341 \\
0.494\end{array}$ \\
\hline $\begin{array}{l}6105 \\
6165 \\
61 \% 5\end{array}$ & 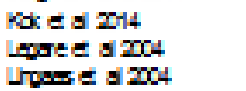 & $\begin{array}{l}0.300 \\
508 \\
0.198\end{array}$ & $\begin{array}{l}0.013 \\
0.241 \\
0.009\end{array}$ & $\begin{array}{r}3.235 \\
106.445 \\
4.114\end{array}$ & $\begin{array}{l}0.494 \\
0.296 \\
0.292\end{array}$ \\
\hline $\begin{array}{l}6105 \\
61 \mathrm{Ex}\end{array}$ & $\begin{array}{l}\text { urde a } 200 \\
\text { Mdax e: a 2011 }\end{array}$ & $\begin{array}{l}0.254 \\
3.122\end{array}$ & $\begin{array}{l}0.010 \\
0.121\end{array}$ & $\begin{array}{r}6539 \\
30391\end{array}$ & $\begin{array}{l}0.408 \\
0.492\end{array}$ \\
\hline $\begin{array}{l}6105 \\
61 \% 5 \\
61 \% 5\end{array}$ & 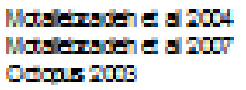 & $\begin{array}{l}0.419 \\
0.315 \\
0.436\end{array}$ & $\begin{array}{l}0.015 \\
0.022 \\
0.044\end{array}$ & $\begin{array}{l}11.007 \\
3074 \\
5.00\end{array}$ & $\begin{array}{l}0.600 \\
0.32 \\
0.55\end{array}$ \\
\hline $\begin{array}{l}6165 \\
61 \% 5 \\
61 \% 5\end{array}$ & $\begin{array}{l}\text { PFACIE } 4 \text { 2004 } \\
\text { ROOBY } 2009 \\
\text { Samane: a } 2003\end{array}$ & $\begin{array}{l}0.133 \\
1.72 \\
1.087\end{array}$ & $\begin{array}{l}0.009 \\
0.72 \\
0.064\end{array}$ & $\begin{array}{r}3.830 \\
4.192 \\
18.408\end{array}$ & $\begin{array}{l}0.274 \\
0.208 \\
0.954\end{array}$ \\
\hline $\begin{array}{l}61 \mathrm{es} \\
61 \mathrm{es}\end{array}$ & B.ART 2003 & $\begin{array}{l}1000 \\
0834\end{array}$ & $\begin{array}{l}0.138 \\
0.52\end{array}$ & $\begin{array}{l}7202 \\
1.48\end{array}$ & $\begin{array}{l}1000 \\
0.64\end{array}$ \\
\hline $\begin{array}{l}66 \pi \\
66 \pi \\
66 \pi\end{array}$ & $\begin{array}{l}\text { Care es a } 2003 \\
\text { COFONARY } 212 \\
\text { lete s } 2003\end{array}$ & $\begin{array}{l}0.333 \\
0.329 \\
0.320\end{array}$ & $\begin{array}{l}0.013 \\
0.511 \\
0.013\end{array}$ & $\begin{array}{l}8.429 \\
1.54 \\
8.25\end{array}$ & $\begin{array}{l}0.506 \\
0.575 \\
0.494\end{array}$ \\
\hline $\begin{array}{l}66 \pi \\
66 \pi\end{array}$ & $\begin{array}{l}\text { Mreedo es a } 2003 \\
\text { Nere ef } 2006\end{array}$ & $\begin{array}{l}0.196 \\
0.829\end{array}$ & $\begin{array}{l}0.009 \\
0.111\end{array}$ & $\begin{array}{l}4.131 \\
4.24\end{array}$ & $\begin{array}{l}0.294 \\
0.699\end{array}$ \\
\hline $\begin{array}{l}66 \pi \\
66 \pi \\
7+\pi\end{array}$ & $\begin{array}{l}\text { Nrope: a } 2006 \\
\text { Do0fs 2012 }\end{array}$ & $\begin{array}{l}1.000 \\
0.301 \\
0.545\end{array}$ & $\begin{array}{l}0.060 \\
0.496 \\
0.249\end{array}$ & $\begin{array}{r}15322 \\
1391 \\
1.196\end{array}$ & $\begin{array}{l}1000 \\
0.395 \\
0.130\end{array}$ \\
\hline$\frac{7+75}{7+\pi 5}$ & $\begin{array}{l}\text { OnOH } 212 \\
\text { FiNGUE-62013 }\end{array}$ & $\begin{array}{l}0.324 \\
0.72\end{array}$ & $\begin{array}{l}0.013 \\
0.118\end{array}$ & $\begin{array}{l}7.984 \\
4.415\end{array}$ & $\begin{array}{l}0.491 \\
0.725\end{array}$ \\
\hline $\begin{array}{l}7+\pi \\
7680 \\
7680\end{array}$ & $\begin{array}{l}\operatorname{ses} 2010 \\
\text { CR }{ }^{2} 214\end{array}$ & $\begin{array}{l}0.58 \\
1.084 \\
2039\end{array}$ & $\begin{array}{l}0.75 \\
0.35 \\
0.179\end{array}$ & $\begin{array}{r}1.120 \\
3.29 \\
23.194\end{array}$ & $\begin{array}{l}0.100 \\
0.89 \\
0.5 \%\end{array}$ \\
\hline $\begin{array}{l}7680 \\
7680\end{array}$ & GOPCAEEZT13 & $\begin{array}{l}0.807 \\
0899\end{array}$ & $\begin{array}{l}0.48 \\
0.58\end{array}$ & $\begin{array}{l}1.361 \\
1399\end{array}$ & $\begin{array}{l}0.421 \\
0.596\end{array}$ \\
\hline Qversl & & $0 \pi 0$ & 0594 & 0.98 & 0.048 \\
\hline
\end{tabular}

Fig. 2 - Forest plot for the incidence of stroke. Cl=confidence intervals

Figure 7 shows the meta-regression plot graphing log OR for myocardial infarction occurrence against the mean age of the patients in the off-pump group. The meta-regression line throughout the graph is close to the line of no effect and the $95 \% \mathrm{Cl}$ are equally dispersed about the line of no effect. Therefore, there is no difference in the modality favoured across the different ages measured and no relationship between age and the $\log \mathrm{OR}(\mathrm{Q}=0.540, P=0.464)$.

\section{DISCUSSION}

A recent editorial comment ${ }^{[6]}$ suggests that it is important to investigate which category of patient would benefit more from either off- or on-pump CABG. One of the ways in which patients can be categorised is according to age, with patient vulnerability increasing with increasing age. In this novel meta-analysis and meta-regression, we have investigated the effect of age on short-term clinical outcomes following off- or on-pump surgery. Most of the results showed that when patients were classified according to 5-year age bands there was no difference in the OR for stroke, mortality, or Ml occurring in the off-pump group compared to the on-pump group. There was a small significant difference in the odds of stroke incidence overall. This was replicated in the meta-regression plots with off-pump favoured for stroke incidence but no differences in the modality favoured according to the different ages measured.

There was no significant difference in the incidence of mortality or MI between on-pump and off-pump CABG overall, mirroring the results of the four largest trials to date $e^{[10,17-19]}$ and the three most recent meta-analyses ${ }^{[2-4]}$. This has been the general trend in many studies to date. In addition to this, there was no evidence from this meta-analysis to suggest that the 


\begin{tabular}{|c|c|c|c|c|c|}
\hline \multirow{2}{*}{$\frac{\text { Group by }}{\text { Age renge }}$} & \multirow[t]{2}{*}{ atudy nams } & \multicolumn{4}{|c|}{ atatistios for sooh study } \\
\hline & & $\begin{array}{l}\text { Odds } \\
\text { ratio }\end{array}$ & $\begin{array}{l}\text { Lower } \\
\text { limit }\end{array}$ & $\begin{array}{c}\text { Upper } \\
\text { IImit }\end{array}$ & p-Valus \\
\hline $51-55$ & lobal e: al 2007 & 0.680 & 0.108 & 4.036 & 0.653 \\
\hline $51-55$ & & 0.660 & 0.108 & 4.096 & 0.653 \\
\hline 5660 & Blcer e: al 2014 & 0.320 & 0.012 & 8.245 & 0.492 \\
\hline 5660 & Gerols e: al 2004 & 0.325 & 0.033 & 3.192 & 0.335 \\
\hline $56-60$ & & 0.323 & 0.050 & 2.096 & 0.236 \\
\hline 6165 & Al-Fumeh e: al 2006 & 3.096 & 0.122 & 75594 & 0.498 \\
\hline 6165 & BHACAS 12002 & 0.196 & 0.009 & 4.135 & 0.295 \\
\hline 6165 & Fatwouch et al 2009 & 0.194 & 0.022 & 1.706 & 0.139 \\
\hline 6165 & Legare e: al 2004 & 2014 & 0.181 & 22.445 & 0.569 \\
\hline 6165 & Lingass e: al 2004 & 1.000 & 0.061 & 16.365 & 1.000 \\
\hline 6165 & Motallebus deh ex al 2004 & 0.419 & 0.016 & 11.027 & 0.602 \\
\hline 6165 & Motallebusdeh e: al 2007 & 1.943 & 0.174 & 21.762 & 0.590 \\
\hline 6165 & OCTOPUS 2003 & 0.979 & 0.136 & 7.046 & 0.983 \\
\hline 6165 & PRAGUE-4 2004 & 1.863 & 0.337 & 10.287 & 0.476 \\
\hline 6165 & Rastan es al 2005 & 3.154 & 0.121 & 82.165 & 0.490 \\
\hline 6165 & Rooby 2009 & 1.385 & 0.675 & 2.840 & 0.375 \\
\hline 6165 & Es himan et al 2003 & 3.383 & 0.131 & 87.112 & 0.452 \\
\hline 6165 & BMART 2003 & 0.495 & 0.044 & 5.548 & 0,568 \\
\hline 6165 & & 1.192 & 0.717 & 1.980 & 0.499 \\
\hline $65-70$ & Camler es al 2003 & 1.032 & 0.062 & $17: 240$ & 0.982 \\
\hline $65-70$ & CORONARY 2012 & 0.867 & 0.610 & 1.231 & 0.425 \\
\hline $65-70$ & Lee et al 2003 & 3.102 & 0.121 & 79208 & 0.494 \\
\hline $65-70$ & Munereso et al 2003 & 1.518 & 0.247 & 9.312 & 0.652 \\
\hline $65-70$ & Nirandan e: al 2006 & 0.325 & 0.013 & 8202 & 0.495 \\
\hline $65-70$ & & 0.889 & 0.634 & 1.247 & 0.495 \\
\hline $71-75$ & Dodes 2012 & 0.873 & 0.314 & 2.428 & 0.795 \\
\hline $71-75$ & $O N-O F F 2012$ & 0.549 & 0.158 & 1.905 & 0.345 \\
\hline $71-75$ & PRAGUEG 2013 & 0.716 & 0.196 & 2.618 & 0.614 \\
\hline $71-75$ & & 0.722 & 0.368 & 1.417 & 0.344 \\
\hline $76-60$ & BBS 2010 & 0.488 & 0.176 & 1.351 & 0.167 \\
\hline $76-80$ & CRISP 2014 & 0.327 & 0.013 & 8.213 & 0.497 \\
\hline $76-60$ & GOPCABE 2013 & 0.907 & 0.554 & 1.496 & 0.699 \\
\hline $76-60$ & & 0.793 & 0.511 & 1.231 & 0.301 \\
\hline Overall & & 0.876 & 0.703 & 1.093 & 0.241 \\
\hline
\end{tabular}

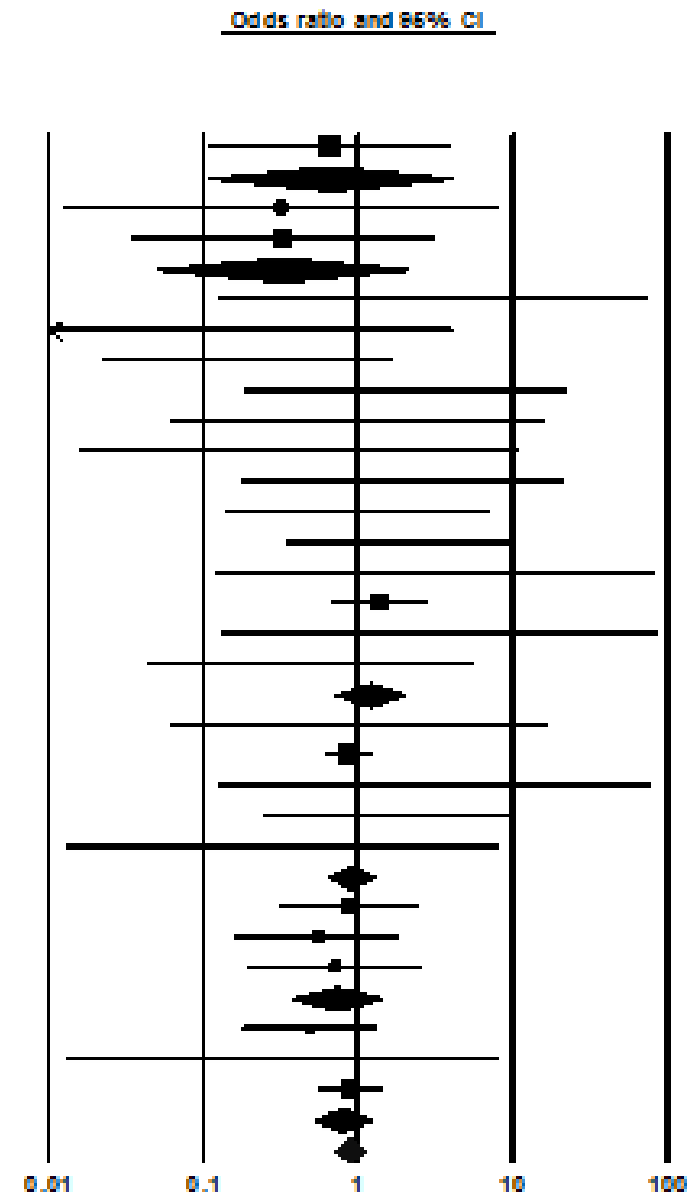

Favours off Favours On

Fig. 3 - Forest plot for the incidence of mortality. Cl=confidence intervals

increasing age influences the occurrence of these outcomes as no significant difference in the meta-regression was found. The single exception to this is the Ml incidence in the 51-55-year age band; however, it should be noted that this result was based on a single trial and clearly more trials investigating this age group are required.

This meta-analysis found a significantly higher occurrence of stroke in the on-pump group overall but no differences in the different age groupings. The overall result concurs with the results of Deppe et al. ${ }^{[3]}$ and Kowalewski et al. ${ }^{[2]}$. However, they contrast with the four largest trials to date $\mathrm{e}^{[10,17-19]}$ and the metaanalysis by Dieberg et al. ${ }^{[4]}$. These findings suggest that if there is a difference in the occurrence of stroke between the off-pump and on-pump groups, then age is not the determining factor. In contrast, other retrospective trials, e.g. Ricci et al. ${ }^{[19]}$ and the meta-analysis by Altarabsheh et al. ${ }^{[1]}$, examined patients older than 80 years and found lower stroke rates in the off-pump CABG patients. There are no RCTs in patients $>80$ years old.
It is often hypothesised that off-pump CABG should produce a lower incidence rate of stroke as it does not involve aortic manipulation and cross-clamping ${ }^{[1]}$. But performing the proximal anastomoses during cross-clamping is one possible solution to reduce the aortic manipulation involved in on-pump CABG, thus weakening this hypothesis. There have been many contradictory results as to whether off-pump CABG reduces the risk of stroke and therefore, a definitive answer has not been reached. This could be due to the occurrence of perioperative stroke during CABG being a relatively rare event, meaning that even large trials and metaanalyses lack the weight to support their results. Nevertheless, it is important to continue this evaluation as stroke is a devastating complication of CABG that can lead to a decreased quality of life and increased mortality rate ${ }^{[20]}$. It is important to link potential preoperative risk factors to the incidence of perioperative stroke in order to improve techniques to reduce its occurrence; however, this meta-analysis suggests that age is not one of them. Another potential risk factor that could be associated with an increased risk of 


\begin{tabular}{|c|c|c|c|c|c|}
\hline \multirow{2}{*}{$\frac{\text { Group by }}{\text { Aqs ra nos }}$} & \multirow[t]{2}{*}{ atudy nams } & \multicolumn{4}{|c|}{ atatistlos for sa oh study } \\
\hline & & $\begin{array}{l}\text { Odds } \\
\text { ratio }\end{array}$ & $\begin{array}{c}\text { Lower } \\
\text { limit }\end{array}$ & $\begin{array}{c}\text { Upper } \\
\text { limit }\end{array}$ & p-Value \\
\hline $51-55$ & lobal et al 2014 & 6.056 & 1.307 & 28.073 & 0.021 \\
\hline $51-55$ & & 6.056 & 1.307 & 28.073 & 0.021 \\
\hline 56.60 & Gerols e: al 2004 & 0.481 & 0.116 & 1.992 & 0.312 \\
\hline $56-60$ & JOCRI 2005 & 1.063 & 0.146 & 7.731 & 0.952 \\
\hline 5680 & Pent:lls e: al 2001 & 1.000 & 0.055 & 18304 & 1.000 \\
\hline 5660 & & 0.670 & 0.229 & 1.962 & 0.465 \\
\hline 6165 & Alfumeh et al 2006 & 1.000 & 0.062 & 16256 & 1.000 \\
\hline 6165 & Asclone a: al 20006 & 3.154 & 0.121 & 82.165 & 0.490 \\
\hline 6165 & BHACAS 12002 & 0.388 & 0.073 & 2.047 & 0.264 \\
\hline 6165 & BHACAS 22002 & 0.667 & 0.109 & 4.078 & 0.661 \\
\hline 6165 & Kahn e: al 2004 & 2.832 & 0.113 & 71.131 & 0.527 \\
\hline 6165 & Legare e: al 2004 & 4.082 & 0.451 & 36.958 & 0.211 \\
\hline 6165 & Llingsess et al 2004 & 3.050 & 0.122 & 76.388 & 0.497 \\
\hline 6165 & Michsux et al 2011 & 1568 & 0.239 & 10.300 & 0.639 \\
\hline 6165 & Oetopus 2003 & 0.749 & 0.271 & 2.070 & 0.577 \\
\hline 6165 & PRAGUE-4 2004 & 1.235 & 0.273 & 5.592 & 0.784 \\
\hline 6165 & Rastan ex al 2005 & 1.000 & 0.058 & 17.181 & 1.000 \\
\hline 6165 & ROOBY 2009 & 0.907 & 0.499 & 1.649 & 0.749 \\
\hline 6165 & Eahlman e: al 2003 & 0.137 & 0.007 & 2.799 & 0.197 \\
\hline 6165 & BMART 2003 & 0.330 & 0.013 & 8.199 & 0.499 \\
\hline 6165 & Vedin e: al 2006 & 3.452 & 0.136 & 87.936 & 0.452 \\
\hline 6165 & & 0.937 & 0.627 & 1.401 & 0.753 \\
\hline $65-70$ & Carrier et al 2003 & 1.032 & 0.062 & 17240 & 0.982 \\
\hline $66-70$ & CORONAFY 2012 & 0.925 & 0.739 & 1.158 & 0.497 \\
\hline $65-70$ & Mun eremo et al 2003 & 0.494 & 0.044 & 5.552 & 0.568 \\
\hline $66-70$ & & 0.921 & 0.737 & 1.151 & 0.469 \\
\hline $71-75$ & DoORs 2010 & 1.523 & 0.901 & 2.575 & 0.116 \\
\hline $71-75$ & On-on 2012 & 0.644 & 0.179 & 2.316 & 0.500 \\
\hline $71-75$ & PRAGUEG 2013 & 0.308 & 0.097 & 0.978 & 0.045 \\
\hline $71-75$ & & 1.078 & 0.689 & 1.688 & 0.742 \\
\hline $76-80$ & BBS 2010 & 0.532 & 0.206 & 1.252 & 0.148 \\
\hline $78-60$ & CRISP 2014 & 1.000 & 0.193 & 5.195 & 1.000 \\
\hline $76-80$ & GOPCABE 2013 & 0.896 & 0.472 & 1.703 & 0.738 \\
\hline $76-80$ & & 0.763 & 0.457 & 1.245 & 0.279 \\
\hline Overall & & 0.937 & 0.795 & 1.105 & 0.439 \\
\hline
\end{tabular}

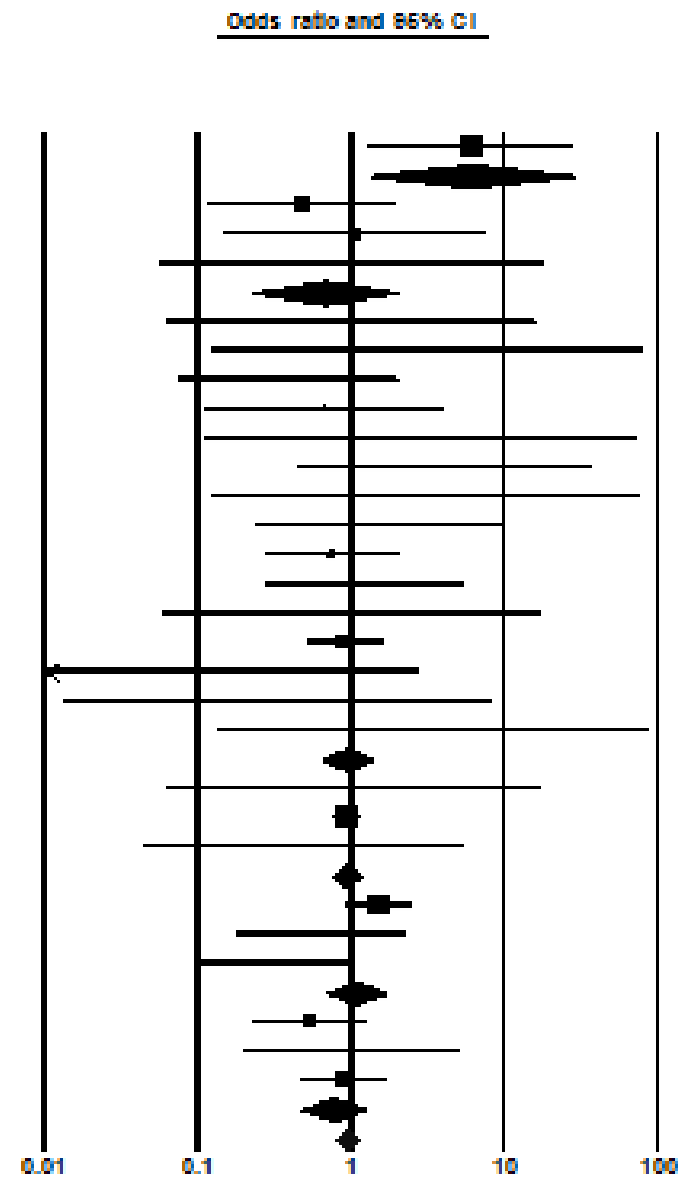

Favours off Favours On

Fig. 4 - Forest plot for the incidence of myocardial infarction (MI). Cl=confidence intervals

stroke is gender. Puskas et al. ${ }^{[21]}$ found that there is a higher incidence of post-operative stroke within the female population, along with a higher mortality and MI rate. They also found that females are more likely to benefit from off-pump CABG than males. Hence, there are many factors that need to be considered and researched further when comparing off-pump and on-pump CABG.

\section{Study Limitations}

Studies scored between two and four out of six on the modified Jadad scale indicating that the median study quality score was moderate (Table 2). There was also some evidence of heterogeneity in many of the studies. Linked to this, not all studies recorded the method of randomisation and there was great variation of methods used between studies. There were also many studies that did not describe dropouts or withdrawals. It is worth noting that it is impossible to use blinding methods within this analysis as surgeons cannot be blinded as to what surgery they are to perform.
One of the most obvious limitations of this study, as in many of the meta-analyses to date that have compared on-pump and off-pump CABG, is the relatively small size of most of the included studies. Only three of the RCTs included more than 1,000 patients $^{[10,17-18]}$ and the next biggest trial included 900 patients ${ }^{[19-22]}$. Many had less than 100 patients (e.9., S4) and some as little as <20 patients (e.g., S6) within their studies. Removing all studies with $<100$ patients did not change the overall results, except for the stroke incidence, where the overall significance disappeared. Moreover, the included studies often reported a low occurrence of events in terms of their clinical endpoints, as previously described. This means that most of the included trials were underpowered and endpoints were underestimated, thus the reliability of their results are affected.

In addition, there are many differences in the methods used in each of the included studies. There is variation in the experience of the surgeons and some studies do not state this. For example, one of the larger studies included in this meta-analysis ${ }^{[17]}$ has 


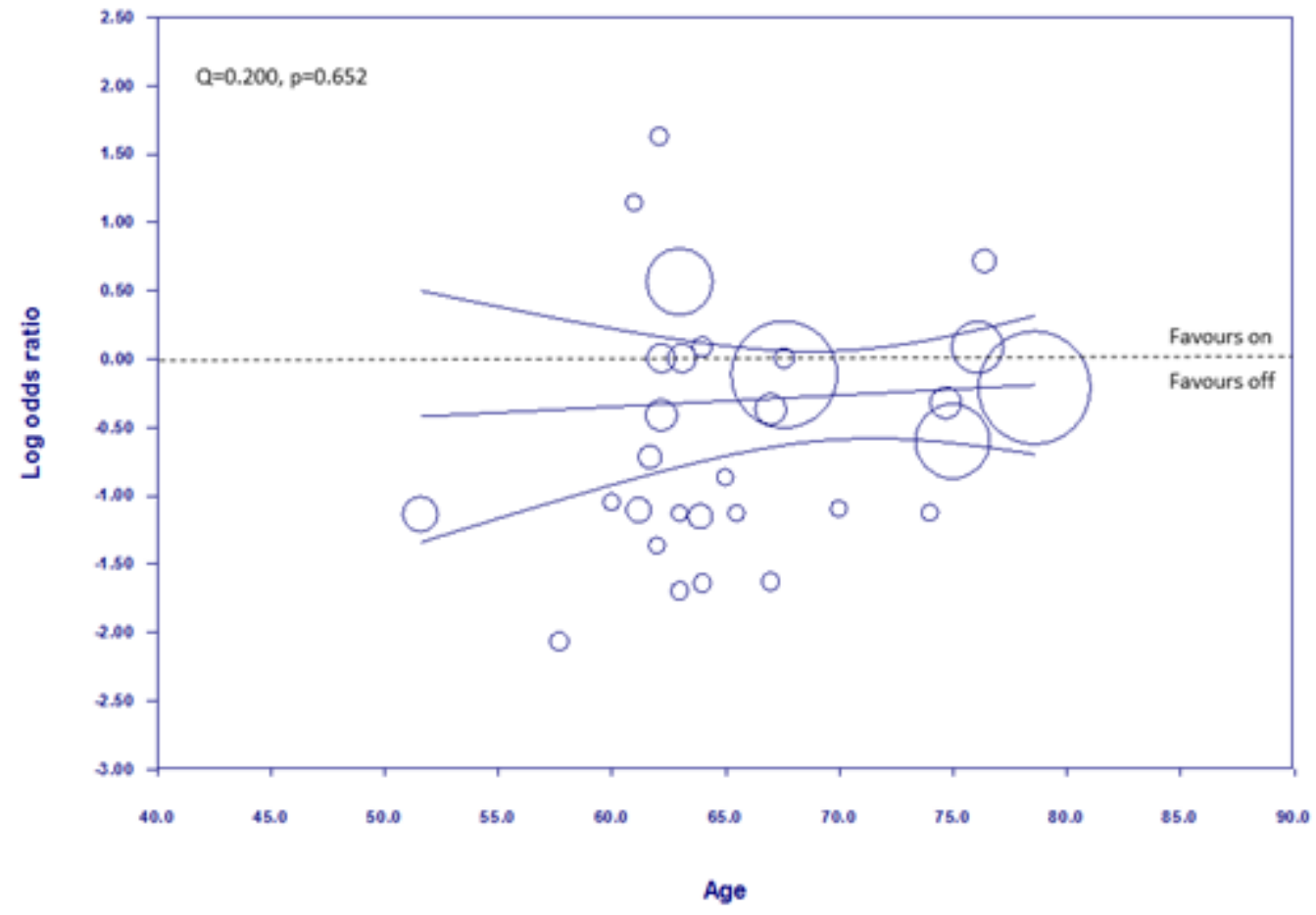

Fig. 5 - Weighted random-effects meta-regression analysis regressing the log odds ratio (OR) of stroke against age in the off-pump group. Negative values of the log OR mean more benefits for stroke associated with off-pump. The size of the circle corresponds to the inverse variance of the $\log O R$, and thus is related to the statistical weight of the individual trial. The curved lines represent the $95 \%$ confidence intervals.

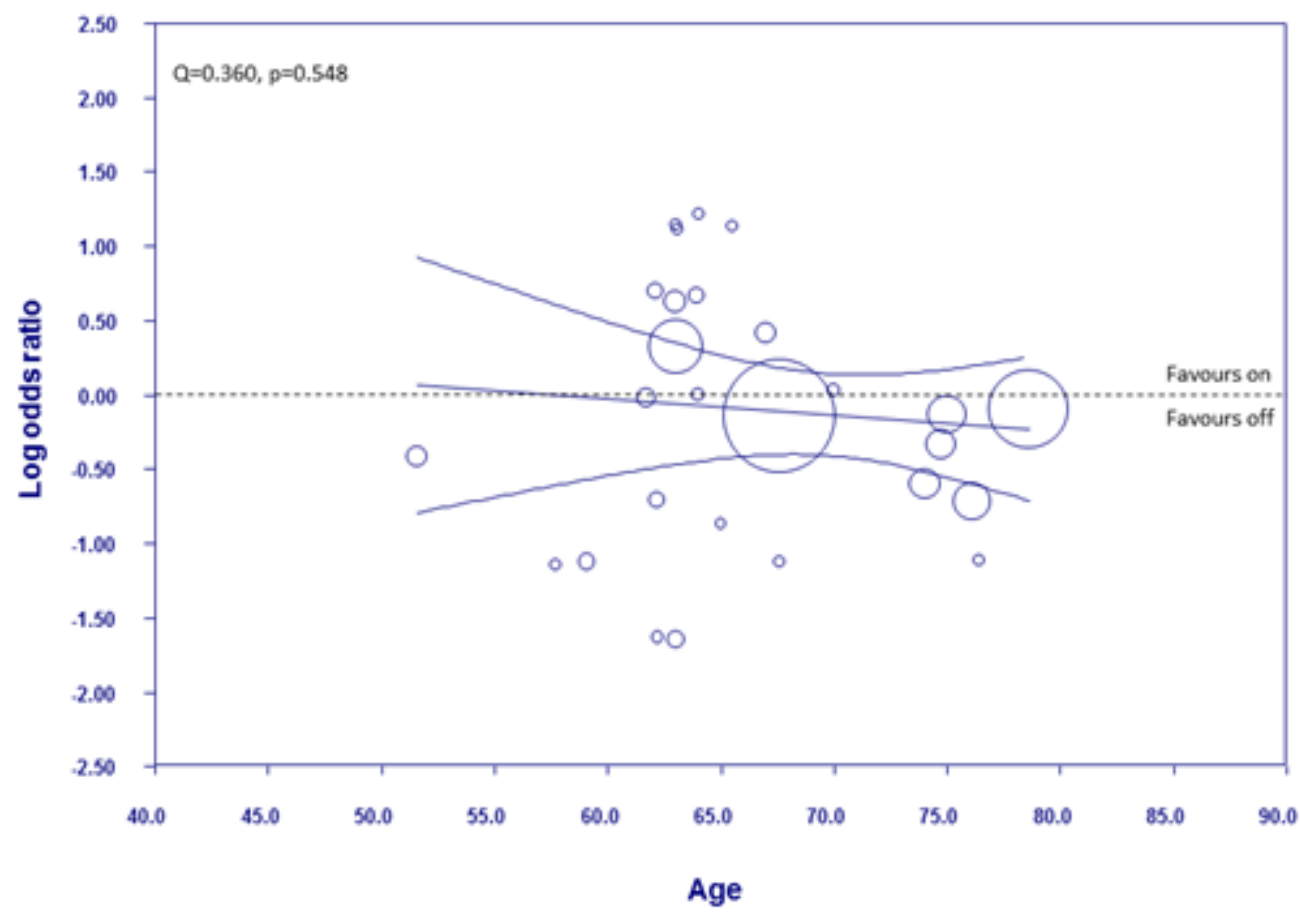

Fig. 6 - Weighted random-effects meta-regression analysis regressing the log odds ratio of mortality against age in the off-pump group. All other details as in Figure 5. 


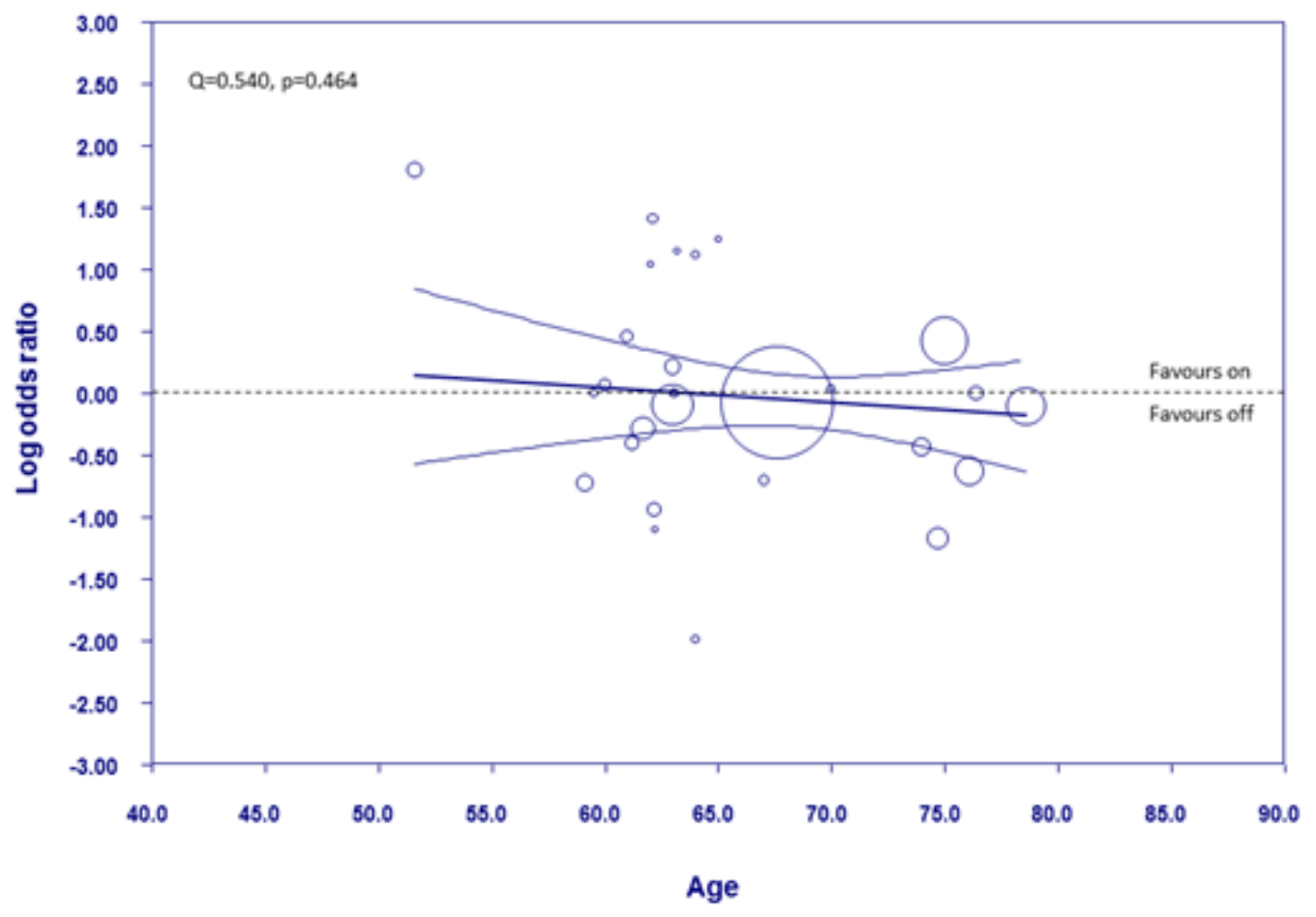

Fig. 7 - Weighted random-effects meta-regression analysis regressing the log odds ratio of myocardial infarction against age in the offpump group. All other details as in Figure 5.

been criticised for the use of trainee surgeons in their trial who were inexperienced in the off-pump CABG procedure. The CABG procedure itself also varied between studies as some surgeons used hypothermic CPB (e.g., S6) whilst others used normothermic CPB (e.g., S7). Similarly, there were some variations in the method of cardioplegic arrest used for on-pump CABG; some trials used cold blood cardioplegia (e.g., S13) and some used warm blood cardioplegia (e.g., S9).

Another big limitation of this study is the small number of trials with a mean age between 51-55 years or $>66$ years, meaning that these age groups were underpowered compared to the others. On top of this, there were no trials with a mean age of over 80 years, meaning that this age group was completely omitted from the analysis. In order to gain a better analysis of the effect that age has on the outcomes of on-pump and off-pump $C A B G$, more trials need to be completed, including patients within these age groups.

\section{CONCLUSION}

There is continuing debate as to which approach on- or off-pump CABG is superior. There are many ways in which patients could be subdivided to discover which selected groups would benefit most from one approach or another, including age. This meta-analysis and meta-regression has shown that separating patients according to their age up to the age of 80 years does not affect whether off-pump or on-pump should be favoured in these patients.

\section{No financial support. \\ No conflict of interest.}

\section{Authors' roles \& responsibilities}

HM Substantial contributions to the conception or design of the work; or the acquisition, analysis, or interpretation of data for the work; final approval of the version to be published.

GD

Substantial contributions to the conception or design of the work; or the acquisition, analysis, or interpretation of data for the work; final approval of the version to be published.

NS Substantial contributions to the conception or design of the work; or the acquisition, analysis, or interpretation of data for the work; final approval of the version to be published.

NK Substantial contributions to the conception or design of the work; or the acquisition, analysis, or interpretation of data for the work; final approval of the version to be published. 


\section{REFERENCES}

1. Apostolakis A, Papakonstantinou NA, Koniari I. Myocardial revascularization without extracorporeal circulation; why hasn't it convinced yet? Ann Card Anaesth. 2017;20(2):219-55. doi:10.4103/aca.ACA_39_16.

2. Kowalewski M, Pawliszak W, Malvindi PG, Bokszanski MP, Perlinski D, Raffa GM, et al. Off-pump coronary artery bypass grafting improves short-term outcomes in high-risk patients compared with on-pump coronary artery bypass grafting: meta-analysis. JThorac Cardiovasc Surg. 2016;151(1):60-77. doi:10.1016/j.jtcvs.2015.08.042.

3. Deppe AC, Arbash W, Kuhn EW, Slottosch I, Scherner M, Liakopoulos OJ, et al. Current evidence of coronary artery bypass grafting off-pump versus on-pump: a systematic review with meta-analysis of over 16,900 patients investigated in randomized controlled trials. Eur J Cardiothorac Surg. 2016;49(4):1031-41. discussion 1041. doi:10.1093/ejcts/ezv268.

4. Dieberg G, Smart NA, King N. On- vs. off-pump coronary artery bypass grafting: a systematic review and meta-analysis. Int J Cardiol. 2016;223:20111. doi:10.1016/j.ijcard.2016.08.250.

5. Smart NA, Dieberg G, King N. Long-term outcomes of on- versus offpump coronary artery bypass grafting. J Am Coll Cardiol. 2018;71(9):983-91. doi:10.1016/j.jacc.2017.12.049.

6. Adams DH, Chikwe J. On-pump CABG in 2018: still the gold standard. J Am Coll Cardiol. 2018;71(9):992-3. doi:10.1016/j.jacc.2018.01.026.

7. Flather M, Rhee JW, Boothroyd DB, Boersma E, Brooks MM, Carrié D, et al. The effect of age on outcomes of coronary artery bypass surgery compared with balloon angioplasty or bare-metal stent implantation among patients with multivessel coronary disease: a collaborative analysis of individual patient data from 10 randomized trials. J Am Coll Cardiol. 2012;60(21):2150-7. doi:10.1016/j.jacc.2012.08.982.

8. Ozen A, Unal EU, Songur M, Kocabeyoglu SS, Hanedan O, Yilmaz M, et al. Coronary artery bypass grafting in the octogenarians: should we intervene, or leave them be? J Geriatr Cardiol. 2015;12(2):147-52. doi:10.11909/j. issn.1671-5411.2015.02.005.

9. Yuksel A, Yolgosteren A, Kan II, Cayir MC, Velioglu Y, Yalcin M, et al. A comparison of early clinical outcomes of off-pump and on-pump coronary artery bypass grafting surgery in elderly patients. Acta Chir Belg. 2018;118(2):99-104. doi:10.1080/00015458.2017.1383087.

10. Diegeler A, Börgermann J, Kappert U, Breuer M, Böning A, Ursulescu A, et al. Off-pump versus on-pump coronary-artery bypass grafting in elderly patients. N Engl J Med. 2013;368(13):1189-98. doi:10.1056/NEJMoa1211666.

11. Altarabsheh SE, Deo SV, Rababa'h AM, Lim JY, Cho YH, Sharma V, et al. Off-pump coronary artery bypass reduces early stroke in octogenarians: a meta-analysis of 18,000 patients. Ann Thorac Surg. 2015;99(5):1568-75. doi:10.1016/j.athoracsur.2014.12.057.

12. Panesar SS, Athanasiou T, Nair S, Rao C, Jones C, Nicolaou M, et al. Early outcomes in the elderly: a meta冈analysis of 4921 patients undergoing coronary artery bypass grafting - comparison between offखpump and on区 pump techniques. Heart. 2006;92(12):1808-16. doi:10.1136/hrt.2006.088450.

13. Zhu ZG, Xiong X, Ding JL, Chen J, LiY, Zhou JL, et al. Comparison of outcomes between off-pump versus on-pump coronary artery bypass surgery in elderly patients: a meta-analysis. Braz J Med Biol Res. 2017;50(3):e5711. doi:10.1590/1414-431X20165711.

14. Moher D, Liberati A, Tetzlaff J, Altman DG. Preferred reporting items for systematic reviews and meta-analyses: the PRISMA statement. Ann Intern Med. 2009;151(4):264-9. doi:10.7326/0003-4819-151-4-200908180-00135.

15. Jadad AR, Moore RA, Carroll D, Jenkinson C, Reynolds DJ, Gavaghan $\mathrm{DJ}$, et al. Assessing the quality of reports of randomized clinical trials: is blinding necessary? Control Clin Trials. 1996;17(1):1-12. doi:10.1016/01972456(95)00134-4.

16. Higgins JP, Altman DG, Gøtzsche PC, Jüni P, Moher D, Oxman AD,et al. The Cochrane collaboration's tool for assessing risk of bias in randomised trials. BMJ. 2011;343:d5928. doi:10.1136/bmj.d5928.

17. Shroyer AL, Grover FL, Hattler B, Collins JF, McDonald GO, Kozora E, et al. On-pump versus off-pump coronary-artery bypass surgery. N Engl J Med. 2009;361(19):1827-37. doi:10.1056/NEJMoa0902905.

18. Lamy A, Devereaux PJ, Prabhakaran D, Taggart DP, Hu S, Paolasso E, et al. Off-pump or on-pump coronary-artery bypass grafting at 30 days. N Engl J Med. 2012;366(16):1489-97. doi:10.1056/NEJMoa1200388.
19. Ricci M, Karamanoukian HL, Abraham R, Von Fricken K, D'Ancona G, Choi $\mathrm{S}$, et al. Stroke in octogenarians undergoing coronary artery surgery with and without cardiopulmonary bypass. Ann Thorac Surg. 2000;69(5):1471-5. doi:10.1016/s0003-4975(00)01238-8.

20. Nicolini F. Stroke after coronary artery bypass grafting: where do we stand? J Heart Stroke. 2017;2(1):1105.

21. Puskas JD, Kilgo PD, Kutner M, Pusca SV, Lattouf O, Guyton RA. Off-pump techniques disproportionately benefit women and narrow the gender disparity in outcomes after coronary artery bypass surgery. Circulation. 2007;116(11 Suppl):1192-9. doi:10.1161/CIRCULATIONAHA.106.678979.

22. Houlind K, Kjeldsen BJ, Madsen SN, Rasmussen BS, Holme SJ, Nielsen PH, et al. On-pump versus off-pump coronary artery bypass surgery in elderly patients: results from the Danish on-pump versus off-pump randomization study. Circulation. 2012;125(20):2431-9. doi:10.1161/CIRCULATIONAHA.111.052571.

23. Iqbal J, Ghaffar A, Shahbaz A, Abid AR. Stroke after coronary artery bypass surgery with and without cardiopulmonary bypass. J Ayub Med Coll Abbottabad. 2014;26(2):123-8.

24. Bicer M, Senturk T, Yanar M, Tutuncu A, Oral AY, Ulukaya E, et al. Effects of off-pump versus on-pump coronary artery bypass grafting: apoptosis, inflammation, and oxidative stress. Heart Surg Forum. 2014;17(5):E271-6. doi:10.1532/HSF98.2014326.

25. Gerola LR, Buffolo E, Jasbik W, Botelho B, Bosco J, Brasil LA, et al. Off-pump versus on-pump myocardial revascularization in low-risk patients with one or two vessel disease: perioperative results in a multicenter randomized controlled trial. Ann Thorac Surg. 2004;77(2):569-73. doi:10.1016/500034975(03)01353-5.

26. Kobayashi J, Tashiro T, Ochi M, Yaku H, Watanabe G, Satoh T, et al. Early outcome of a randomized comparison of off-pump and on-pump multiple arterial coronary revascularisation. Circulation. 2005;112(9 Suppl):1338-43. doi:10.1161/CIRCULATIONAHA.104.524504.

27. Penttilä HJ, Lepojärvi MV, Kiviluoma KT, Kaukoranta PK, Hassinen IE, Peuhkurinen KJ. Myocardial preservation during coronary surgery with and without cardiopulmonary bypass. Ann Thorac Surg. 2001;71 (2):565-71. doi:10.1016/s0003-4975(00)02002-6.

28. Al-Ruzzeh S, George S, Bustami M, Wray J, IIsley C, Athanasiou T, et al. Effect of off-pump coronary artery bypass surgery on clinical, angiographic, neurocognitive, and quality of life outcomes: randomised controlled trial. BMJ. 2006;332(7554):1365. doi:10.1136/bmj.38852.479907.7C.

29. Angelini GD, Taylor FC, Reeves BC, Ascione R. Early and midterm outcome after off-pump and on-pump surgery in beating heart against cardioplegic arrest studies (BHACAS 1 and 2): a pooled analysis of two randomised controlled trials. Lancet 2002;359(9313):1194-9. doi:10.1016/S01406736(02)08216-8.

30. Ascione R, Caputo M, Calori G, Lloyd CT, Underwood MJ, Angelini GD. Predictors of atrial fibrillation after conventional and beating heart coronary surgery: a prospective, randomized study. Circulation. 2000;102(13):1530-5. doi:10.1161/01.cir.102.13.1530.

31. Fattouch K, Guccione F, Dioguardi P, Sampognaro R, Corrado E, Caruso M, et al. Off-pump versus on-pump myocardial revascularization in patients with ST-segment elevation myocardial infarction: a randomized trial. J Thorac Cardiovasc Surg. 2009;137(3):650-6; discussion 656-7. doi:10.1016/j. jtcvs.2008.11.033.

32. Jongman RM, Zijlstra JG, KokWF, van Harten AE, Mariani MA, Moser J, et al. Off-pump CABG surgery reduces systemic inflammation compared with on-pump surgery but does not change systemic endothelial responses: a prospective randomized study. Shock. 2014;42(2):121-8. doi:10.1097/ SHK.0000000000000190.

33. Khan NE, De Souza A, Mister R, Flather M, Claque J, Davies S, et al. A randomized comparison of off-pump and on-pump multivessel coronary-artery bypass surgery. N Engl J Med. 2004;350(1):21-8. doi:10.1056/NEJMoa031282.

34. Kok WF, van Harten AE, Koene BM, Mariani MA, Koerts J, Tucha O, et al. A pilot study of cerebral tissue oxygenation and postoperative cognitive dysfunction among patients undergoing coronary artery bypass grafting randomised to surgery with or without cardiopulmonary bypass. Anaesthesia. 2014;69(6):613-22. doi:10.1111/anae.12634.

35. Légaré JF, Buth KJ, King S, Wood J, Sullivan JA, Hancock Friesen C, et al. Coronary bypass surgery performed off pump does not result in lower in- 
hospital morbidity than coronary artery bypass grafting performed on pump. Circulation. 2004:109(7):887-92. doi:10.1161/01.CIR.0000115943.41814.7D.

36. Lingaas PS, Hol PK, Lundblad R, Rein KA, Tønnesen TI, Svennevig JL, et al. Clinical and angiographic outcome of coronary surgery with and without cardiopulmonary bypass: a prospective randomized trial. Heart Surg Forum. 2004;7(1):37-41.

37. Lund C, Hol PK, Lundblad R, Fosse E, Sundet K, Tennøe B, et al. Comparison of cerebral embolization during off-pump and on-pump coronary artery bypass surgery. Ann Thorac Surg. 2003;76(3):765-70; discussion 770. doi:10.1016/ s0003-4975(03)00679-9.

38. Michaux I, Filipovic M, Skarvan K, Bolliger D, Schumann R, Bernet F, et al. A randomized comparison of right ventricular function after on-pump versus off-pump coronary artery bypass graft surgery. J Thorac Cardiovasc Surg. 2011;141(2):361-7. Erratum in: J Thorac Cardiovasc Surg. 2011;142(3):726. doi:10.1016/j.jtcvs.2010.02.023.

39. Motallebzadeh R, Kanagasabay R, Bland JM, Kaski JC, Jahangiri M. S100 protein and its relation to cerebral microemboli in on-pump and off-pump coronary artery bypass surgery. Eur J Cardiothorac Surg. 2004;25(3):409-14. doi:10.1016/j.ejcts.2003.12.018

40. Motallebzadeh R, Bland JM, Markus HS, Kaski JC, Jahangiri M. Neurocognitive function and cerebral emboli: randomized study of on-pump versus offpump coronary artery bypass surgery. Ann Thorac Surg. 2007;83(2):475-82. doi:10.1016/j.athoracsur.2006.09.024.

41. Nathoe HM, van Dijk D, Jansen EW, Suyker WJ, Diephuis JC, van Boven WJ, et al. A comparison of on-pump and off-pump coronary bypass surgery in lowrisk patients. N Engl J Med. 2003;348(5):394-402. doi:10.1056/NEJMoa021775.

42. Puskas JD, Williams WH, Duke PG, Staples JR, Glas KE, Marshall JJ, et al. Offpump coronary artery bypass grafting provides complete revascularization with reduced myocardial injury, transfusion requirements, and length of stay: a prospective randomized comparison of two hundred unselected patients undergoing off-pump versus conventional coronary artery bypass grafting. J Thorac Cardiovasc Surg. 2003;125(4):797-808. doi:10.1067/mtc.2003.324.

43. Rastan AJ, Bittner HB, Gummert JF, Walther T, Schewick CV, Girdauskas E, et al. On-pump beating heart versus off-pump coronary artery bypass surgery-evidence of pump-induced myocardial injury. Eur J Cardiothorac Surg. 2005;27(6):1057-64. doi:10.1016/j.ejcts.2005.03.007

44. Sahlman A, Ahonen J, Nemlander A, Salmenperä M, Eriksson H, Rämö J, et al. Myocardial metabolism on off-pump surgery; a randomized study of 50 cases. Scand Cardiovasc J. 2003;37(4):211-5. doi:10.1080/14017430310001726.

45. Shroyer AL, Grover FL, Hattler B, Collins JF, McDonald GO, Kozora E, et al. On-pump versus off-pump coronary-artery bypass surgery. N Engl J Med. 2009;361(19):1827-37. doi:10.1056/NEJMoa0902905.

46. Straka Z, Widimsky P, Jirasek K, Stros P, Votava J, VanekT, et al. Off-pump versus on-pump coronary surgery: final results from a prospective randomized study PRAGUE-4. Ann Thorac Surg. 2004;77(3):789-93. doi:10.1016/j. athoracsur.2003.08.039.

47. Vedin J, Nyman H, Ericsson A, Hylander S, Vaage J. Cognitive function after on or off pump coronary artery bypass grafting. Eur J Cardiothorac Surg. 2006;30(2):305-10. doi:10.1016/j.ejcts.2006.03.037.

48. Carrier M, Perault LP, Jeanmart H, Martineau R, Cartier R, Page P. Randomized trial comparing off-pump to on-pump coronary artery bypass grafting in high risk patients. Heart Surg Forum. 2003;6(6):E89-92.

49. Lamy A, Devereaux PJ, Prabhakaran D, Taggart DP, Hu S, Paolasso E, et al. Off-pump or on-pump coronary-artery bypass grafting at 30 days. N Engl J Med. 2012;366(16):1489-97. doi:10.1056/NEJMoa1200388.

50. Lee JD, Lee SJ, Tsushima WT, Yamauchi H, Lau WT, Popper J, et al. Benefits of off-pump bypass on neurologic and clinical morbidity: a prospective randomized trial. Ann Thorac Surg. 2003;76(1):18-25; discussion 25-6. doi:10.1016/s0003-4975(03)00342-4.

51. Muneretto C, Bisleri G, Negri A, Manfredi J, Metra M, Nodari S, et al. Offpump coronary artery bypass surgery technique for total arterial myocardial revascularization: a prospective randomized study. Ann Thorac Surg. 2003;76(3):778-82; discussion 783. doi:10.1016/s0003-4975(03)00564-2.

52. Nesher N, Frolkis I, Vardi M, Sheinberg N, Bakir I, Caselman F, et al. Higher levels of serum cytokines and myocardial tissue markers during on-pump versus off-pump coronary artery bypass surgery. J Card Surg. 2006;21 (4):395402. doi:10.1111/j.1540-8191.2006.00272.x.

53. Niranjan G, Asimakopoulos G, Karagounis A, Cockerill G, Thompson M, Chandrasekaran V. Effects of cell saver autologous blood transfusion on blood loss and homologous blood transfusion requirements in patients undergoing cardiac surgery on- versus off-cardiopulmonary bypass: a randomised trial. Eur J Cardiothorac Surg. 2006;30(2):271-7. doi:10.1016/j. ejcts.2006.04.042.

54. Hlavicka J, Straka Z, Jelinek S, et al. PPRAGUE-6 trial: off-pump versus onpump coronary artery bypass graft surgery in patients with EuroSCORE 6 . Prague: Third Faculty of Medicine, Charles University Prague; 2013 [cited 2019 Jul 21]. Available from: http://clinicaltrialresults.org/Slides/ACC\%20 2013/Hlavicka_PRAGUE-6_ACC\%202013.pdf

55. Houlind K, Kjeldsen BJ, Madsen SN, Rasmussen BS, Holme SJ, Nielsen PH, et al. On-pump versus off-pump coronary artery bypass surgery in elderly patients: results from the Danish on-pump versus off-pump randomization study. Circulation. 2012;125(20):2431-9. doi:10.1161/CIRCULATIONAHA.111.052571.

56. Lemma MG, Coscioni E, Tritto FP, Centofanti P, Fondacone C, Salica A, et al. On-pump versus off-pump coronary artery bypass surgery in high-risk patients: operative results of a prospective randomized trial (on-off study). J Thorac Cardiovasc Surg. 2012;143(3):625-31. doi:10.1016/j.jtcvs.2011.11.011.

57. Møller CH, Perko MJ, Lund JT, Andersen LW, Kelbaek H, Madsen JK, et al. No major differences in 30-day outcomes in high-risk patients randomized to offpump versus on-pump coronary bypass surgery: the best bypass surgery trial. Circulation. 2010;121(4):498-504. doi:10.1161/CIRCULATIONAHA.109.880443.

58. Rogers CA, Pike K, Campbell H, Reeves BC, Angelini GD, Gray A, et al. Coronary artery bypass grafting in high-RISk patients randomised to off- or on-pump surgery: a randomised controlled trial (the CRISP trial). Health Technol Assess. 2014;18(44):V-XX,1-157. doi:10.3310/hta18440.

Supplementary Fig. 1 - Pubmed Search Strategy.

\#1 SEARCH "cardiopulmonary bypass"[MeSH Terms] OR ("cardiopulmonary"[All Fields]

AND "bypass"[All Fields]) OR "cardiopulmonary bypass"[All Fields]

\#2 SEARCH "coronary artery bypass"[MeSH Terms] OR ("coronary"[All Fields] AND

"artery"[All Fields] AND "bypass"[All Fields]) OR "coronary artery bypass"[All Fields] OR

("coronary"[All Fields] AND "artery"[All Fields] AND "bypass"[All Fields] AND "grafting"[All

Fields]) OR "coronary artery bypass grafting"[All Fields]

\#3 SEARCH "off pump" [All Fields]

\#4 SEARCH "on pump" [All fields]

\#5 SEARCH \#1 AND \#2

\#6 SEARCH \#3 OR \#4

\#7 SEARCH \#5 AND \#6 
Supplementary Table 1. Excluded studies and reasons for exclusion.

\begin{tabular}{|c|c|}
\hline Study & Reason \\
\hline Chowdhury et al., 2008 & Mean age crossed over two age groups \\
\hline Covino et al., 2001 & Did not record mean age \\
\hline Formica et al., 2013 & Mean age crossed over two age groups \\
\hline Gulielmos et al., 2000 & Mean age crossed over two age groups \\
\hline Hernandez et al., 2007 & Did not record mean age \\
\hline Hoel et al., 2007 & Did not record mean age \\
\hline Kobayashi et al., 2005 & Mean age crossed over two age groups \\
\hline Kochamba et al., 2000 & Mean age crossed over two age groups \\
\hline Kunes et al., 2007 & Mean age crossed over two age groups \\
\hline Medved et al., 2008 & Mean age crossed over two age groups \\
\hline Paparella et al., 2006 & Did not record mean age \\
\hline Rachwalik et al., 2006 & Mean age crossed over two age groups \\
\hline Rainio et al., 2007 & Mean age crossed over two age groups \\
\hline Raja et al., 2003 & Did not record mean age \\
\hline
\end{tabular}

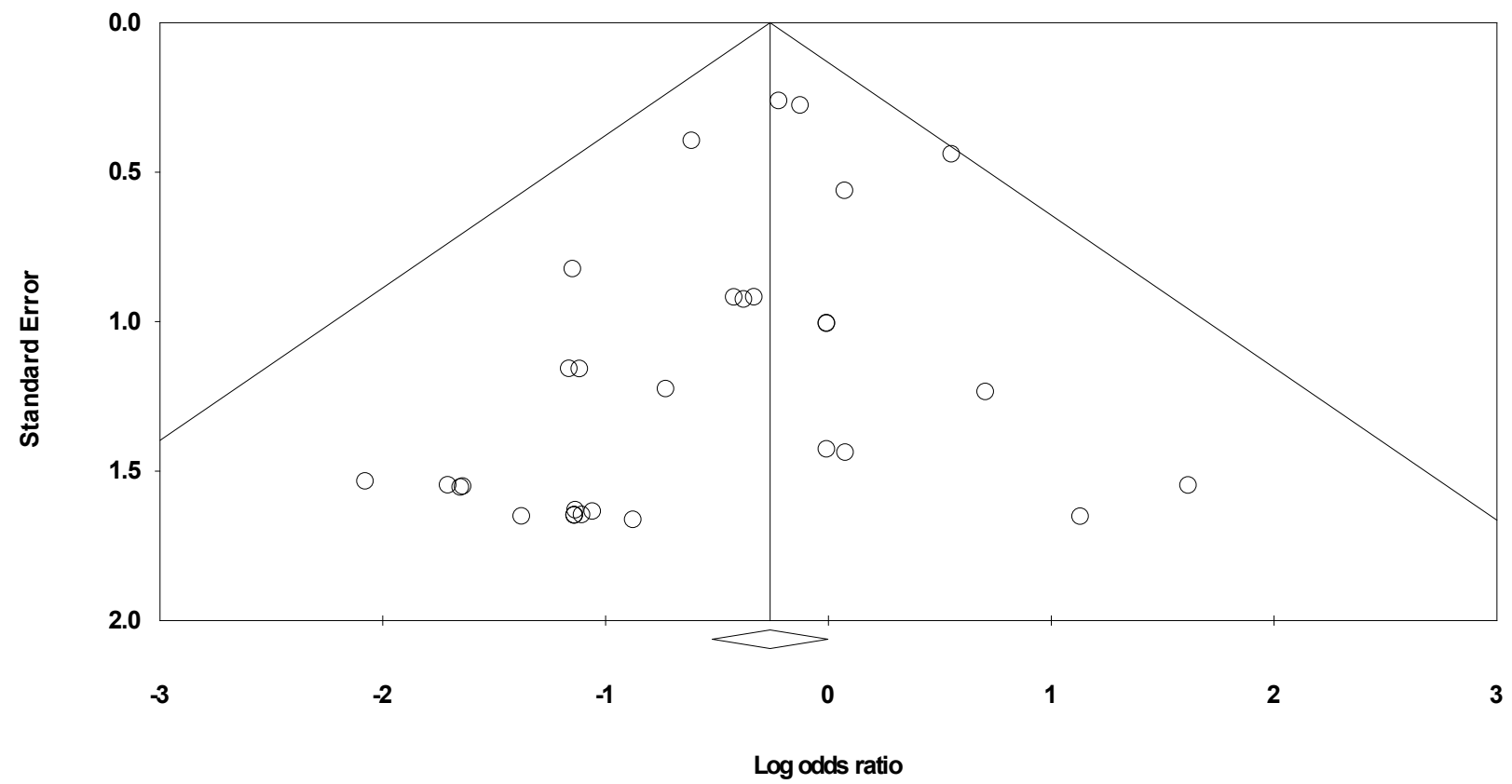

Supplementary Fig. 2 - Funnel plot for the incidence of stroke. Begg and Mazumdar's test (P value): 0.262 and Egger's test (P value): 0.031. 


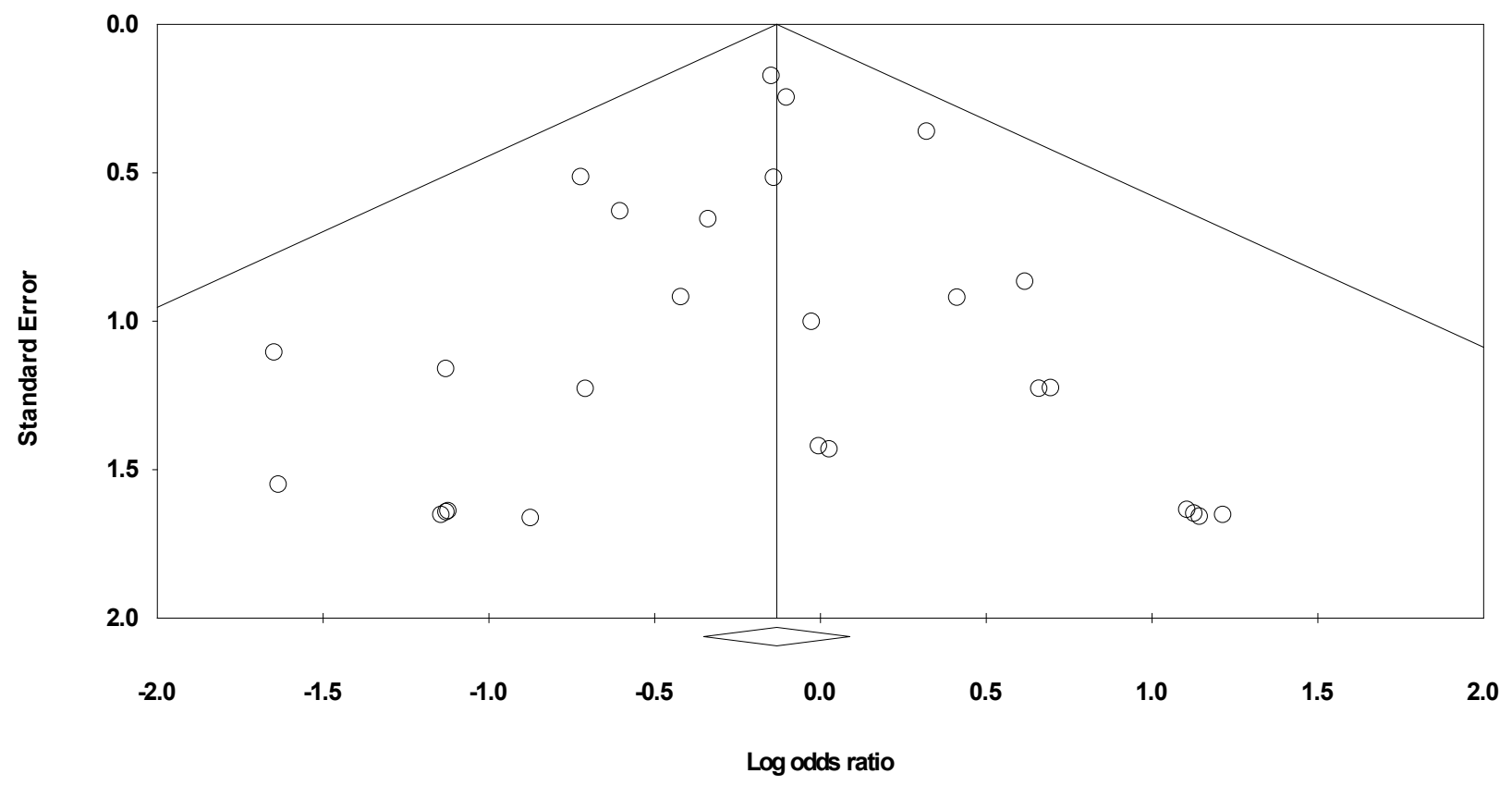

Supplementary Fig. 3 - Funnel plot for the incidence of mortality. Begg and Mazumdar's test (P value): 0.692 and Egger's test (P value): 0.736.

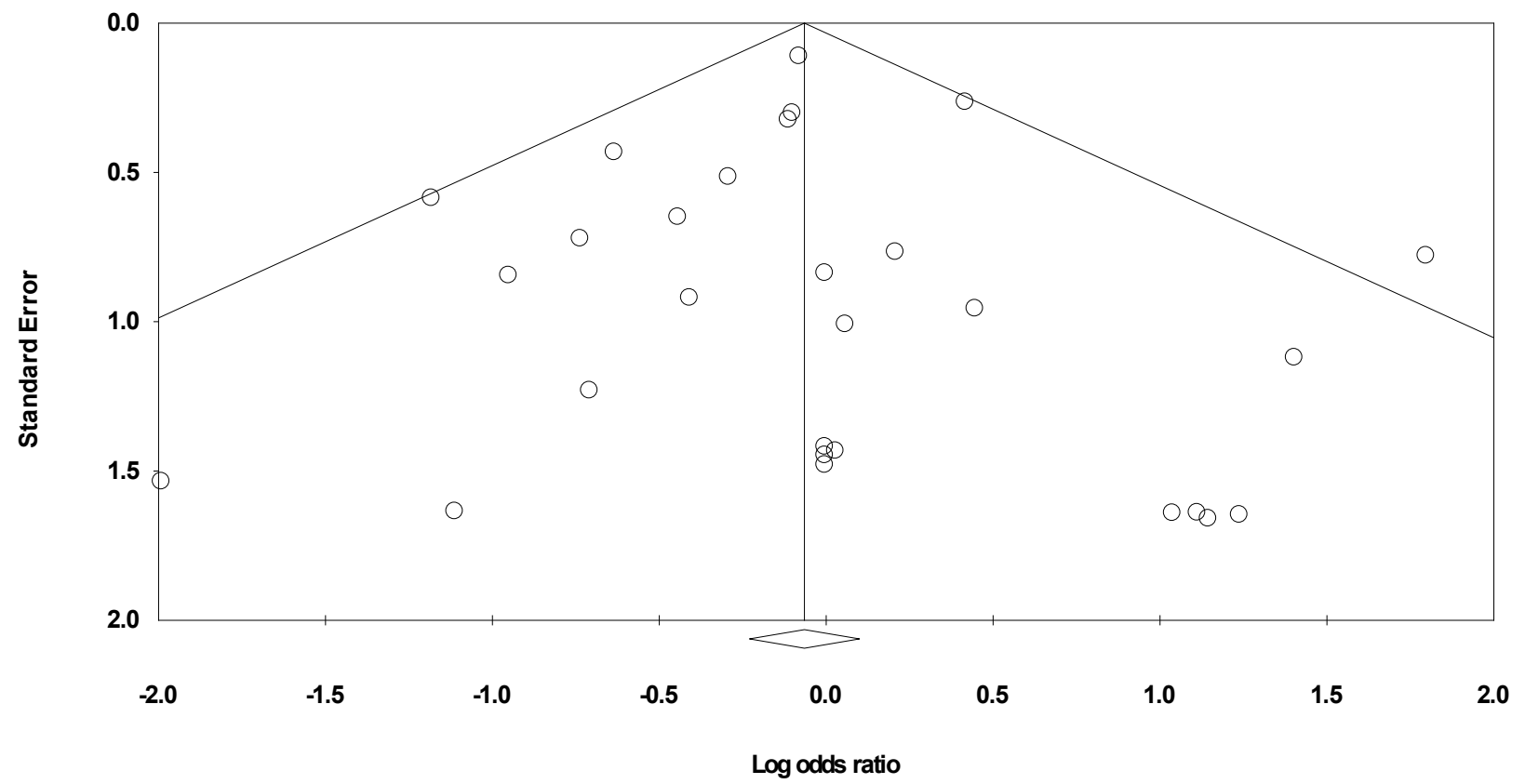

Supplementary Fig. 4 - Funnel plot showing the incidence of myocardial infarction. Begg and Mazumdar's test (Pvalue): 0.167 and Egger's test (P value): 0.903. 
Supplementary Table 2 . Examination of study quality.

\begin{tabular}{|c|c|c|c|c|c|c|c|}
\hline Study & Randomisation & $\begin{array}{l}\text { Methods of } \\
\text { randomisation }\end{array}$ & $\begin{array}{l}\text { Methods } \\
\text { of blinding } \\
\text { described }\end{array}$ & $\begin{array}{l}\text { Method of } \\
\text { blinding } \\
\text { appropriate }\end{array}$ & $\begin{array}{c}\text { Withdrawals/ } \\
\text { dropouts } \\
\text { described }\end{array}$ & $\begin{array}{c}\text { Other } \\
\text { potential } \\
\text { bias }\end{array}$ & $\begin{array}{c}\text { Score } \\
\text { (out of 6) }\end{array}$ \\
\hline Al-Ruzzeh et al. ${ }^{[28]}, 2006$ & Yes & Yes & No & $\mathrm{N} / \mathrm{A}$ & No & No & 3 \\
\hline Angelini et al. ${ }^{[29]}, 2002$ & Yes & Yes & No & $\mathrm{N} / \mathrm{A}$ & No & Yes & 2 \\
\hline Ascione et al. ${ }^{[30]}, 2000$ & Yes & Yes & No & $\mathrm{N} / \mathrm{A}$ & No & No & 3 \\
\hline Bicer et al. ${ }^{[24]}, 2014$ & Yes & No & No & $\mathrm{N} / \mathrm{A}$ & No & No & 2 \\
\hline Carrier et al. ${ }^{[48]}, 2003$ & Yes & No & No & $\mathrm{N} / \mathrm{A}$ & No & No & 2 \\
\hline Diegeler et al. ${ }^{[10]}, 2013$ & Yes & Yes & No & $\mathrm{N} / \mathrm{A}$ & Yes & No & 4 \\
\hline Fattouch et al. ${ }^{[31]}, 2009$ & Yes & Yes & No & $\mathrm{N} / \mathrm{A}$ & No & No & 3 \\
\hline Gerola et al. ${ }^{[25]}, 2004$ & Yes & No & No & $\mathrm{N} / \mathrm{A}$ & No & No & 2 \\
\hline Hlavicka et al. ${ }^{[54]}, 2013$ & Yes & Yes & No & $\mathrm{N} / \mathrm{A}$ & Yes & No & 4 \\
\hline Houlind et al. ${ }^{[22]}, 2012$ & Yes & Yes & No & $\mathrm{N} / \mathrm{A}$ & Yes & No & 4 \\
\hline lqbal et al. ${ }^{[23]}, 2014$ & Yes & No & No & $\mathrm{N} / \mathrm{A}$ & No & No & 2 \\
\hline Jongman et al. [32], 2014 & Yes & No & No & $\mathrm{N} / \mathrm{A}$ & No & No & 2 \\
\hline Khan et al. ${ }^{[33]}, 2004$ & Yes & No & No & $\mathrm{N} / \mathrm{A}$ & No & No & 2 \\
\hline Kobayashi et al. ${ }^{[26]}, 2005$ & Yes & Yes & No & $\mathrm{N} / \mathrm{A}$ & No & No & 3 \\
\hline Kok et al. ${ }^{[34]}, 2014$ & Yes & No & No & N/A & Yes & No & 3 \\
\hline Lamy et al..49], 2012 & Yes & Yes & No & N/A & No & No & 3 \\
\hline Lee et al. ${ }^{[50]}, 2003$ & Yes & Yes & No & $\mathrm{N} / \mathrm{A}$ & No & No & 3 \\
\hline Légaré et al. [35], 2004 & Yes & Yes & No & $\mathrm{N} / \mathrm{A}$ & No & Yes & 2 \\
\hline Lemma et al. ${ }^{[56]}, 2012$ & Yes & Yes & No & $\mathrm{N} / \mathrm{A}$ & Yes & No & 4 \\
\hline Lingaas et al. ${ }^{[36]}, 2004$ & Yes & No & No & $\mathrm{N} / \mathrm{A}$ & No & No & 2 \\
\hline Lund et al. ${ }^{[37]}, 2003$ & Yes & No & No & $\mathrm{N} / \mathrm{A}$ & Yes & No & 3 \\
\hline Michaux et al. ${ }^{[38]}, 2011$ & Yes & Yes & No & $\mathrm{N} / \mathrm{A}$ & No & Yes & 2 \\
\hline Moller et al. ${ }^{[57]}, 2010$ & Yes & Yes & No & $\mathrm{N} / \mathrm{A}$ & No & Yes & 2 \\
\hline Motallebzadeh et al. ${ }^{[39]}, 2004$ & Yes & Yes & No & $\mathrm{N} / \mathrm{A}$ & No & No & 3 \\
\hline Motallebzadeh et al. ${ }^{[40]}, 2007$ & Yes & Yes & No & $\mathrm{N} / \mathrm{A}$ & No & No & 3 \\
\hline Munereto et al. ${ }^{[51]}, 2003$ & Yes & No & No & $\mathrm{N} / \mathrm{A}$ & No & No & 2 \\
\hline Nathoe et al. ${ }^{[41]}, 2003$ & Yes & Yes & No & $\mathrm{N} / \mathrm{A}$ & No & No & 3 \\
\hline Nesher et al..52], 2006 & Yes & Yes & No & $\mathrm{N} / \mathrm{A}$ & Yes & No & 4 \\
\hline Niranjan et al. ${ }^{[53]}, 2006$ & Yes & Yes & No & $\mathrm{N} / \mathrm{A}$ & No & No & 3 \\
\hline Penttila et al. ${ }^{[27]}, 2001$ & Yes & No & No & $\mathrm{N} / \mathrm{A}$ & No & No & 2 \\
\hline Puskas et al. ${ }^{[42]}, 2003$ & Yes & Yes & No & N/A & Yes & Yes & 3 \\
\hline Rastan et al. ${ }^{[43]}, 2005$ & Yes & Yes & No & $\mathrm{N} / \mathrm{A}$ & No & No & 3 \\
\hline Rogers et al. ${ }^{[58]}, 2014$ & Yes & Yes & No & $\mathrm{N} / \mathrm{A}$ & Yes & Yes & 3 \\
\hline Sahlman et al. ${ }^{[44]}, 2003$ & Yes & No & No & $\mathrm{N} / \mathrm{A}$ & No & No & 2 \\
\hline Shroyer et al. ${ }^{[45]}, 2009$ & Yes & Yes & No & $\mathrm{N} / \mathrm{A}$ & Yes & Yes & 3 \\
\hline Straka et al. ${ }^{[46]}, 2004$ & Yes & Yes & No & $\mathrm{N} / \mathrm{A}$ & Yes & No & 4 \\
\hline Vedin et al. ${ }^{[47]}, 2006$ & Yes & No & No & $\mathrm{N} / \mathrm{A}$ & Yes & No & 3 \\
\hline
\end{tabular}

Median score $=3$. N/A=not available 